Efficiency and Voluntary Implementation in Markets with Repeated Pairwise Bargaining Author(s): Matthew O. Jackson and Thomas R. Palfrey

Source: Econometrica, Vol. 66, No. 6 (Nov., 1998), pp. 1353-1388

Published by: Econometric Society

Stable URL: http://www.jstor.org/stable/2999620

Accessed: 08-03-2016 00:40 UTC

Your use of the JSTOR archive indicates your acceptance of the Terms \& Conditions of Use, available at http://www.jstor.org/page/ info/about/policies/terms.jsp

JSTOR is a not-for-profit service that helps scholars, researchers, and students discover, use, and build upon a wide range of content in a trusted digital archive. We use information technology and tools to increase productivity and facilitate new forms of scholarship. For more information about JSTOR, please contact support @jstor.org. 


\title{
EFFICIENCY AND VOLUNTARY IMPLEMENTATION IN MARKETS WITH REPEATED PAIRWISE BARGAINING
}

\author{
By Matthew O. Jackson and Thomas R. Palfrey ${ }^{1}$
}

\begin{abstract}
We examine a simple bargaining setting, where heterogeneous buyers and sellers are repeatedly matched with each other. We begin by characterizing efficiency in such a dynamic setting, and discuss how it differs from efficiency in a centralized static setting. We then study the allocations which can result in equilibrium when the matched buyers and sellers bargain through some extensive game form. We take an implementation approach, characterizing the possible allocation rules which result as the extensive game form is varied. We are particularly concerned with the impact of making trade voluntary: imposing individual rationality on and off the equilibrium path. No buyer or seller consumates an agreement which leaves them worse off than the discounted expected value of their future rematching in the market. Finally, we compare and contrast the efficient allocations with those that could ever arise as the equilibria of some voluntary negotiation procedure.
\end{abstract}

KEYWORDS: Bargaining, implementation, matching.

\section{INTRODUCTION}

THIS PAPER USES implementation theory to study decentralized contracting in markets that are limited to bilateral bargaining. To this end, we employ a simple model of matching and search with an infinity of buyers and sellers, who wish to trade one (indivisible) unit of a good. There is a known distribution of seller and buyer valuations. Trade occurs in a finite number of discrete periods. In the first period, buyers and sellers are randomly matched into pairs and then play a bargaining game that either results in a trade at some price, or no trade. If a buyer-seller match does not result in a trade, then each is randomly rematched with a new potential trading partner in the next period. The cost of search comes from each agent having only a finite number of opportunities to trade and discounting between periods. We characterize the efficient allocations and identify the set of allocations that can be achieved by general bargaining procedures.

Our main departure from past work in this area is that we approach the problem from the implementation theory perspective. On the one hand, consistent with much of the previous literature on decentralized bilateral trade, the

\footnotetext{
${ }^{1}$ This project was initiated while Jackson was visiting the California Institute of Technology and continued while he was at Northwestern University and while Palfrey was visiting CREST-LEI and CERAS; we are grateful for their support. We are also grateful for financial support provided under NSF Grant SBR-9507912. We thank Nabil Al-Najjar, Larry Ausubel, Eddie Dekel, Ray Deneckere, Larry Jones, Dilip Mookherjee, Mike Peters, Larry Samuelson, and Asher Wolinsky for helpful conversations and suggestions. We have benefited from the careful comments and suggestions of an editor and three anonymous referees on an earlier draft.
} 
matching and search technology described above is taken as given. But contrary to past work on decentralized bilateral trade, we do not treat the rules of trade as exogenously fixed. That is, our objective is not to study properties of equilibria under some specific game form according to which bilateral trade is governed (say, the Rubinstein bargaining game, or the Nash bargaining solution), but rather to pose the implementation question: what allocation rules can be implemented as equilibrium outcomes of some finite extensive form bargaining game of perfect information?

We first characterize the set of efficient allocation rules in this environment, and then characterize the set of all allocation rules that can be implemented by some bargaining game form. Using the conditions for implementability, we show that there exist robust distributions of buyer and seller valuations in which efficient trading rules cannot be implemented by any bargaining game. Implementation requires that the desired set of allocations coincide exactly with the set of equilibrium outcomes. In fact, we show the stronger statement that efficient trading rules are not even attainable by any bargaining game, where attainability only requires that an efficient trading rule correspond to some equilibrium outcome.

The characterization of efficient allocations subject to the matching constraint identifies systematic distortions relative to unconstrained efficiency. The unconstrained first best is to have a match result in trade if and only if the buyer has a value above the competitive equilibrium price trade and the seller has a value below that price. (The transaction price is irrelevant.) Subject to matching, however, first best will generally be unachievable since chance determines which buyers are matched with which sellers. As a result, constrained efficiency can involve trade between buyers and sellers whose values both fall below (or above) the competitive equilibrium price. We show that the constrained efficient allocations are uniquely determined (up to sets of measure zero) and characterize such allocations.

We then investigate the implementability of those constrained efficient rules by a general class of finite-length extensive game forms with perfect information. Also, the game form is augmented by appending to each terminal node a signature move for both the buyer and the seller. Both signatures are required, or the mechanism results in no trade for that match. The role of the signatures is to ensure that trade is voluntary, i.e. respects (endogenous) individual rational: ity constraints. It is assumed that the the buyer and seller in the match have complete information about each others' valuations, so the solution concept we employ is effectively backward induction.

In addition to the general characterization, we demonstrate the importance of the implementation approach by showing an example where efficient trades are not attainable when prices correspond to those from Nash Bargaining, but are attainable when prices correspond to Nash Bargaining with a price cap. Finally, based on some of the necessary conditions from the characterizations, we provide a robust example with heterogeneous seller and buyer valuations where 
the efficient allocations are not implementable or even attainable, even if there is no discounting.

\section{RELATION TO THE LITERATURE}

Because this paper bridges several different areas, we discuss separately how it fits in with previous work in three broad themes: competitive bargaining, search, and implementation. In short, what we are doing here is layering the implementation question on to a standard model of search and competitive bargaining. Thus, our work relates to each of these areas.

\section{Relation to the Competitive Bargaining Literature}

The underlying model that we study involves a combination of matching, bargaining, search, and rematching over a sequence of trading periods. As such, it is useful, for studying pure exchange economies from a noncooperative, game-theoretic perspective. Past work in the area ${ }^{2}$ has typically assumed both the technological features underlying the matching and search technologies and also has assumed the formal rules according to which bargaining between paired agents is required to follow. It is this latter set of assumptions that marks the first key difference between what we are doing and what has been done before. While the bargaining rules usually are modeled as a specific process of offers and counteroffers such as one based on Rubinstein (1982) and Stahl (1972), we explicitly do not assume a particular game form for the bargaining process. Rather, we are trying to identify the set of allocation rules (Walrasian or otherwise) that can be achieved as unique Nash equilibrium outcomes of some bargaining mechanism.

The second difference between this paper and earlier work is that we do not focus on the question of the equivalence between Walrasian and competitive bargaining outcomes when market frictions are small. In fact, our main focus is not the case of frictionless markets per se, but rather on the properties of markets in which frictions exist, despite the large numbers of traders. To this end, we characterize efficient allocation rules subject to the matching constraints, and show how these differ in systematic and interesting ways from competitive allocations. Our interest then turns to whether these efficient allocations can be attained via any bargaining rules.

\footnotetext{
${ }^{2}$ By now the collection of papers in this area is too large to summarize exhaustively. The most closely related papers include Gale (1986a, b), Rubinstein and Wolinsky (1985), Binmore and Herrero (1988), and McClennan and Sonnenschein (1991) which follow in the footsteps of the early work on search and matching by Butters (1980), Mortensen (1982), Diamond (1982), and others. The bulk of this work is interested in identifying conditions under which game-theoretic equilibria in these decentralized matching and bilateral bargaining institutions will approximate Walrasian allocations when the frictions (search costs, discount factors, etc.) become infinitesimal. We lump all these together under the general heading of "competitive bargaining."
} 


\section{Relation to the Search Literature}

Sattinger (1995) studies the question of efficiency in a search model with two-sided heterogeneity. ${ }^{3} \mathrm{He}$ finds that the equilibria of matching procedures in which trades take place at prices determined by the Nash bargaining solution can be inefficient even taking account of the constraints of the search process. That is, one cannot even attain "second best" efficiency. ${ }^{4}$ The reason for inefficiency in Sattinger's model is that agents who are faced with a choice of trading in a current match do not account for the effect that their choice has on the future distribution of valuations in the market, and thus the future value from matching of other agents. ${ }^{5}$ There is a problem of congestion and the prices determined by the Nash bargaining solution do not generate adequate incentives for trade to compensate for this externality. In particular, agents do not consummate some trades that society would like them to. The innovation of our work is to investigate arbitrary bargaining procedures and ask whether any such procedure can be constructed to provide agents with the correct incentives for trading. To do this, we characterize the entire set of pricing and allocation rules that can be implemented by some bargaining procedure and compare this set to the set of constrained efficient allocations. The previous work in this area assumes Nash bargaining to determine transaction prices. We demonstrate that in some environments, this kind of pricing is suboptimal since it creates adverse incentive problems which can easily be avoided by resorting to alternative trading mechanisms.

Specifically, we provide an example where the efficient trading rule is not attained when prices correspond to Nash bargaining, but can be attained by a simple variation where prices correspond to Nash bargaining with a price cap. Thus, inefficiency under Nash bargaining is not necessarily evidence that efficiency is not attainable. However, we go on to show that there are examples where efficiency is not attainable via any bargaining procedure. Actually, this example identifies a different source of inefficiency that is complementary to the congestion problem identified in Sattinger (1995) (and Shimer and Smith (1994)). There, agents are too patient and pass up efficiency-enhancing trades. In our example, some agents are overly impatient given their anticipated prospects for trade under any mechanism and so they trade too soon. This reduces the future prospects for other agents below the socially efficient level and creates further impatience.

\footnotetext{
${ }^{3}$ Also related are papers by Lu and McAffee (1995) and Peters (1991) who study the allocation rules generated by specific processes of noncooperative competitive bargaining constrained by matching and Ponsati and Sakovics (1995) who study bargaining with outside options. Shimer and Smith $(1994,1996)$ study this matching problem and obtain additional results and characterizations about efficient sorting subject to the constraints of the matching process and the Nash bargaining solution.

${ }^{4}$ This contrasts with earlier work of Mortensen (1982) and Hosios (1990) who showed that efficiency could be achieved, but in models with homogeneous agents.

${ }^{5}$ We thank an anonymous referee for directing us to the Sattinger (1995) and Shimer and Smith (1994) papers.
} 


\section{Relation to the Implementation Literature}

Implementation theory formally models trading mechanisms as game forms and tries to obtain general characterizations of the allocation rules that can or cannot be achieved as noncooperative equilibrium outcomes. Although the necessary conditions that come out of this literature must be taken seriously, there is somewhat less consensus about the practicality of many of the sufficiency results, where very general and abstract mechanisms are constructed in order to demonstrate that a certain class of allocation rules can be implemented. The canonical mechanisms have been criticized for a variety of reasons rèlating to their artificiality, reliance on threats, discontinuities, lack of balance, lack of well defined behavior on parts of the mechanisms. ${ }^{6}$

In this paper, we want to avoid the problems of artificiality as well as the problems inherent in mechanisms for which behavior is not always well-defined relative to the solution concept. In addition, we wish to begin to remedy two other shortcomings of the existing work in implementation theory.

First, we wish to avoid the use of implausible threats, used either to enforce certain actions in equilibrium, or to prevent certain strategy profiles from being "undesirable" equilibria. An extreme example of such a threat (which appears often in sufficiency constructions) is for the planner to destroy all or part of the social endowment, if a particular out-of-equilibrium message profile is announced. The problem with this is that such outcomes may not actually be carried out, and agents should anticipate this when deciding on strategies. Such mechanisms seem particularly far-fetched in cases where the players have inherent property rights (such as an initial endowment or outside option) that provide a lower bound on the utility the agent can expect in the mechanism, for all message profiles. In our model, because the buyer and seller in a match will be rematched in the next period, should they fail to agree to exchange, this places a natural individual rationality, or voluntary participation, constraint on the process: no buyer or seller will consummate a trade that leaves him or her worse off than the discounted expected value of their future rematching in the market. ${ }^{7}$ We call this voluntary implementation.

Voluntary implementation is related to implementation in the face of renegotiation since renegotiation also provides agents with an option outside of what is immediately prescribed by the mechanism. For example, the approach in Maskin and Moore (1987) is to specify an arbitrary, exogenous, and state dependent

\footnotetext{
${ }^{6}$ There ia a growing literature related to these points and some representative references for various aspects of the problem are: Postlewaite and Wettstein (1989), Jackson (1992), Abreu and Matsushima (1992), Dutta, Sen, and Vohra (1995), Saijo, Tatamitani, and Yamato (1993), Jackson, Palfrey, and Srivastava (1994), and Sjöström (1995).

${ }^{7}$ See Ma, Moore, and Turnbull (1988) for a look at implementation with an exogenous outside option for each player. In our paper, individual rationality is more involved since voluntary implementation takes the form of an endogenous individual rationality constraint that is determined by the value of future rematching, which in turn depends on the bargaining mechanism itself.
} 
renegotiation function that converts inefficient outcomes into efficient ones. ${ }^{8}$ In contrast, our approach does not allow agents to negotiate outside of the explicit rules of the mechanism, so there is no "renegotiation" per se. In particular, we consider finite horizon mechanisms where one or both of the agents may opt for "no trade," effectively walking away from the current match, after which there is no further interaction between those two agents. Given this available option for no trade (which except at the last date leads to rematching), no mechanism can impose an outcome of trade between two matched agents. However, given that agents cannot negotiate outside of a mechanism, it is possible for a mechanism to impose an outcome of no trade between two matched agents even when those two agents have mutual gains from current trade.

As for any process of renegotiation, we use the mechanism to represent whatever the protocol for negotiation between the parties is. Our viewpoint in this paper is thus different from the usual implementation "planner imposes a mechanism" viewpoint. Instead our point of view is more positive in that the full interaction between any agents including any renegotiation that they might undertake can be modeled as a game form. Thus, any interaction between the agents is a process that can be described in full by a game form, and any distinction between negotiation and renegotiation becomes a question of semantics. Moreover, after this full process has concluded, the outcome is not final until both agents have signed a piece of paper acknowledging any agreement that they have reached.

Our approach imposes restrictions that (i) the whole process of interaction can be modeled as a finite length game, and (ii) the process itself is not state dependent. Point (i) is inessential to our results (see footnote 30) and used for simplification. Point (ii) represents an important difference between our approach and that of, say, Maskin and Moore (1987). The set of available means for negotiation (i.e., the mechanism) is the language, pieces of paper, and timing, etc., available for interaction between agents. These same means are available regardless of the preferences of two matched agents. What differs is what agents choose to do as it depends on the state (their preferences, match, time, etc.). We think it is essential that the renegotiation process be formally modeled as part of the game form, and be independent of the state (although the actions chosen may be state dependent). This is consistent with the seminal work on mechanism design by Hurwicz (1972).

We should add that this approach will have some important implications for examples of markets where the option for agents to walk away from a current match is present. For instance, considering the U.S. market for single family homes, there is a standard process of negotiation by which a price is posted, and

\footnotetext{
${ }^{8}$ Rubinstein and Wolinsky (1992) adopt a different approach, "renegotiation-proof implementation," which requires Pareto efficiency of the continuation outcome at all outcome nodes of the implementing mechanism. A related constraint is "credibility," or the inability to commit to off-equilibrium-path outcomes that the planner (as opposed to the players) would not wish to impose. See, for example, Chakravorti, Corchon, and Wilkie (1992), Baliga, Corchon, and Sjöström (1997), and Baliga and Sjöström (1995).
} 
then offers and counter offers are made, lawsuits are brought, escrow accounts are impounded, etc. - and these are the same set of available actions that any further negotiation or "renegotiation" also follow, and constitute an overall game form. Any tentative agreement is not binding until the proper signatures are put to paper. The same is true in many security markets (e.g. NASDAQ or the Chicago Mercantile Exchange) and in fact, most of these exchanges prohibit negotiation between member parties outside of the given rules (i.e. mechanisms) for trade.

The second issue where we depart from past work in implementation theory is to study dynamic allocation rules. The importance of intertemporal tradeoffs is critical since many problems in which economists are interested, such as bargaining, investment, and growth, are dynamic. Unfortunately, implementation theory thus far has had little to contribute to questions of mechanism design in this large arena. Extensive form games have been examined, but only in the context of using them to implement static allocations. ${ }^{9}$ Finally, we emphasize that the notion of implementation we examine here is stronger than simple implementation by subgame perfect equilibrium. Our implementation results are for mechanisms that are constructed as games of perfect information, so our concept of equilibrium is actually "backward induction" (as in Herrero and Srivastava (1992)).

Summarizing our contributions relative to the implementation literature: using a competitive bargaining model with rematching, we are able to characterize implementability in a dynamic environment, with an endogenous voluntary participation constraint, and without imposing implausible threats or using mechanisms with artificial or suspicious features. Thus, we obtain a characterization of what is implementable in this class of dynamic allocation problems, without resorting to the usually cumbersome methods of proof in implementation theory.

\section{Remarks on the Information Structure}

In our model, agents know the value of the agent with whom they are currently matched and there is a central authority who enforces the rules of the mechanism independent of any knowledge of the values of the agents. This assumption is common to each of the literatures discussed above, as well as the contract theory literature. ${ }^{10}$ This approach permits the analysis of mechanism design to focus on incentive problems without introducing the complications of prior beliefs, strategic information transmission, and Bayesian equilibrium. Clearly, most bargaining settings involve some asymmetry of information between negotiating buyers and sellers, and such asymmetric information further compounds the incentive problems and introduces additional potential sources

\footnotetext{
${ }^{9}$ Two recent exceptions are Kalai and Ledyard (1995) and Brusco and Jackson (1996).

${ }^{10}$ This literature is too large to survey here. For example, see Hart and Moore (1988), Moore (1992), Aghion, Dewatripont, and Ray (1994), and the references they cite.
} 
of inefficiency. Our choice is to use a model with symmetric information between bargainers and to focus on a particular source of social inefficiency that arises independently of asymmetric information. From a practical standpoint, in many markets, including some real estate and specialized labor markets, informational asymmetries may play a relatively small role compared to the fundamental problems of value-specific matching and negotiation on which we focus.

The remainder of the paper is organized as follows. The model and definitions are presented in Section 3. Constrained efficient allocation rules are characterized in Section 4. Section 5 provides characterizations of voluntary attainability and voluntary implementability. Section 6 combines the results of Sections 4 and 5 to study the implementation of constrained efficient allocation rules. Section 7 contains some concluding remarks.

\section{DEFINITIONS}

\section{The Economy}

There are two goods. One good is indivisible and the other is divisible. Each seller is endowed with one unit of the indivisible good, and each buyer is endowed with one unit of the divisible (numeraire) good.

\section{Preferences}

Agents' preferences are characterized by a reservation value of the indivisible good, $v \in[0,1]$. There are a finite number of dates, $t \in\{1, \ldots, T\}$, at which trade can take place, and a common discount parameter $\delta \in[0,1]$. A seller with reservation value $s$ who sells her indivisible good for $p$ units of the numeraire good at time $t$ receives (net) utility $\delta^{t}(p-s)$, and a buyer with reservation value $b$ who buys a unit of the indivisible good for $p$ units of the numeraire good at time $t$ receives utility $\delta^{t}(b-p)$. An agent who never trades receives utility 0 .

\section{Distributions of Values}

Initially, there is a continuum of buyers and of sellers. The distribution of reservation values of the agents remaining in the economy at the beginning of a time $t \in\{1, \ldots, T\}$ is summarized by the following functions.

$B_{t}(b)$ - the mass of buyers at time $t$ with value no more than $b$;

$S_{t}(s)$ - the mass of sellers at time $t$ with value no more than $s$.

These are not cumulative distribution functions, since, for instance, it may be that $S_{t}(1) \neq 1$. The corresponding distribution functions (for $S_{t}(1)>0$ and $B_{t}(1)$ $>0)$ are $S_{t}(v) / S_{t}(1)$ and $B_{t}(v) / B_{t}(1)$. The initial mass of buyers and sellers is the same, $B_{1}(1)=S_{1}(1)$, so it will always be true that $B_{t}(1)=S_{t}(1)$, for all $t$. This 
is without loss of generality, since we can model other cases by adding buyers or sellers who should never trade. ${ }^{11}$

We assume that at least one of the two distributions is atomless. Specifically, we will assume that the initial distribution of buyers, $B_{1}$, is continuous and increasing at all $b>0$. This rules out masses of buyers with identical valuations and assures that there are buyers with values in any open subinterval of $[0,1]$. This assumption simplifies the analysis in that we do not have to worry about rationing agents with the same valuation, or randomizing. We also assume that $S_{1}(0)<S_{1}(1)$, to rule out the trivial case where all matches should be consummated immediately in the first period.

\section{Pairwise Matching}

At the beginning of each period, the remaining buyers and sellers who have not yet traded are pairwise matched with each other. The matching ${ }^{12}$ is described by a probability measure $\mu_{t}$ on $[0,1]^{2}$ where for any measurable $A_{t} \subset[0,1]^{2}$

$$
\mu_{t}\left(A_{t}\right)=\int_{s}\left(\int_{b:(s, b) \in A_{t}} \frac{d B_{t}(b)}{B_{t}(1)}\right) \frac{d S_{t}(s)}{S_{t}(1)} .
$$

The distribution over values with which any seller with valuation $s$ will be matched at time $t$ is $d B_{t}(b) / B_{t}(1)$. Similarly, the distribution over values with which any specific buyer with valuation $b$ will be matched at time $t$ is $d S_{t}(s) / S_{t}(1)$.

Matched buyers and sellers are fully informed of each other's valuation.

\section{Allocation Rules}

Allocation rules describe which buyers and sellers will trade at each time, and what price will be paid (i.e., what transfer is made). We restrict our attention to allocation rules that depend only on the time and on the buyers' and sellers' valuations (but not their names). This restriction reflects our interest in anonymous processes.

A trading rule is a collection, $A=\left(A_{1}, \ldots, A_{T}\right)$, of measurable subsets $A_{t}$ of $[0,1] \times[0,1]$. A pair $(s, b) \in A_{t}$ indicates that any seller with valuation $s$ and buyer with valuation $b$ who are matched at time $t$ should trade.

\footnotetext{
${ }^{11}$ For instance, $B_{1}(1)>S_{1}(1)$, is handled by adding sellers with $s=1$.

${ }^{12}$ There is a measurability problem associated with a law of large numbers over a continuum of i.i.d. random variables (see Judd (1985) and Feldman and Gilles (1985)). For any finite economy which approximates ours, we could describe a matching process (which would not be i.i.d.) with the above specified properties, but there would necessarily be some (small) dependence in the random variables. Instead, we work directly at the limit distributions and simply note that we could come arbitrarily close to finding a matching process that formally justifies the assumed one. See Gretsky, Ostroy, and Zame (1992) and Al-Najjar (1996) for more discussion of this.
} 
A price rule is a collection of measurable functions $p=\left(p_{1}, \ldots, p_{T}\right)$, where $p_{t}: A_{t} \rightarrow[0,1]$. A price rule indicates that if a buyer and seller trade, then the buyer transfers $p_{t}(s, b)$ units of the divisible good to the seller.

An allocation rule consists of a trading rule and a price rule.

\section{Cutoff Rules}

One type of trading rule that will play an important role in our results is a cutoff rule. This is a rule such that the set of buyers who trade with any given seller form an upper interval of the set of buyer types, and the set of sellers who trade with a given buyer form a lower interval of the set of seller types. More formally, $A$ is a cutoff rule if for all $t$ and $s,{ }^{13}$

(i) either $\left\{b \mid(s, b) \in A_{t}\right\}=\left\{b \in[0,1] \mid b \geq b^{\prime}\right\}$ or $\left\{b \mid(s, b) \in A_{t}\right\}=\{b \in[0,1] \mid b>$ $\left.b^{\prime}\right\}$ for some $b^{\prime} \in[0,1]$, and

(ii) $\left\{b \mid(s, b) \in A_{t}\right\} \subset\left\{b \mid\left(s^{\prime}, b\right) \in A_{t}\right\}$ whenever $s>s^{\prime}$.

In many cases it will not matter whether the inequalities in (i) are weak or strict (see the definition of equivalence below), and we represent a cutoff rule by functions $\beta_{t}(s)$ (corresponding to $b^{\prime}$ in (i)).

\section{Evolution of Distributions of Valuations}

Any trading rule $A$ and initial distributions $S_{1}$ and $B_{1}$ induce $S_{2}, \ldots, S_{T}$ and $B_{2}, \ldots, B_{T}$, according to the matching process. The resulting distributions are defined recursively by

$$
S_{t+1}(v)=S_{t}(v)-\int_{s \leq v}\left(\int_{b:(s, b) \in A_{t}} \frac{d B_{t}(b)}{B_{t}(1)}\right) d S_{t}(s)
$$

and

$$
B_{t+1}(v)=B_{t}(v)-\int_{b \leq v}\left(\int_{s:(s, b) \in A_{t}} \frac{d S_{t}(s)}{S_{t}(1)}\right) d B_{t}(b) .
$$

\section{Equivalence of Trading and Allocation Rules}

Given the continuum of agents, we define an equivalence over allocation rules that differ only on sets of measure 0 .

The trading rules $A$ and $\hat{A}$ are equivalent if $\mu_{t}\left(A_{t} \cap \hat{A}_{t}\right)=\mu_{t}\left(A_{t} \cup \hat{A}_{t}\right)$ for each $t$, where $\mu_{t}$ is the measure defined in (0) induced by $A$ according to (1) and (2). ${ }^{14}$

The allocation rules $(A, p)$ and $(\hat{A}, \hat{p})$ are equivalent if $A$ and $\hat{A}$ are equivalent and $\mu_{t}\left(\left\{(s, b) \in A_{t} \mid p_{t}(s, b) \neq \hat{p}_{t}(s, b)\right\}\right)=0$ for each $t$.

\footnotetext{
${ }^{13}$ The definition can equivalently be stated from the buyer's perspective.

${ }^{14}$ Notice that in this case the measure $\hat{\mu}_{t}$ induced by $\hat{A}_{t}$ will coincide with $\mu_{t}$.
} 


\section{Expected Utility}

The expected utility $u_{t}^{s}(s ; A, p)$ of a seller with valuation $s$ under an allocation rule $(A, p)$ at the beginning of time $t$ conditional on not having traded yet is given by

$$
\begin{aligned}
u_{t}^{s}(s ; A, p)= & \sum_{\tau=1}^{T} \delta^{\tau-t}\left(\prod_{i=t}^{\tau-1}\left[1-\int_{b:(s, b) \in A_{i}} \frac{d B_{i}(b)}{B_{i}(1)}\right]\right) \\
& \times\left(\int_{b:(s, b) \in A_{\tau}}\left(p_{\tau}(s, b)-s\right) \frac{d B_{\tau}(b)}{B_{\tau}(1)}\right),
\end{aligned}
$$

where $\prod_{i=t}^{t-1}[\cdot]$ is taken to be 1 . Similarly, the expression for the expected utility $u_{t}^{b}(b, A, p)$ of a buyer with valuation $b$ under an allocation rule $(A, p)$ is given by

$$
\begin{aligned}
u_{t}^{b}(b, A, p)= & \sum_{\tau=t}^{T} \delta^{\tau-t}\left(\prod_{i=t}^{\tau-1}\left[1-\int_{s:(s, b) \in A_{i}} \frac{d S_{i}(s)}{S_{i}(1)}\right]\right) \\
& \times\left(\int_{v_{s}:(s, b) \in A_{\tau}}\left(b-p_{\tau}(s, b)\right) \frac{d S_{\tau}(s)}{S_{\tau}(1)}\right) .
\end{aligned}
$$

\section{Reservation Prices}

It will often be useful to work with the reservation prices, $\bar{p}_{t}^{s}(s ; A, p)$ and $\bar{p}_{t}^{b}(b ; A, p)$, induced by an allocation rule. The reservation price at time $t$ is simply the price at which an individual would be indifferent between trading and not trading at time $t$. These follow immediately from above:

$$
\begin{array}{ll}
\bar{p}_{t}^{s}(s ; A, p)-s=\delta u_{t+1}^{s}(s ; A, p) & (t=1, \cdots, T-1), \\
b-\bar{p}_{t}^{b}(b ; A, p)=\delta u_{t+1}^{b}(b ; A, p) & (t=1, \cdots, T-1), \\
\bar{p}_{T}^{s}(s ; A, p)=s, & \\
\bar{p}_{T}^{b}(b ; A, p)=b . &
\end{array}
$$

When $(A, p)$ is fixed, we may simply write $u_{t}^{s}(s), \bar{p}_{t}^{s}(s)$, etc.

\section{Constrained Efficiency}

We say that a trading rule $A$ is constrained efficient if there exists a price rule $p$ such that $(A, p)$ maximizes the total expected surplus:

$$
\int_{s} u_{1}^{s}(s ; A, p) d S_{1}(s)+\int_{b} u_{1}^{b}(b ; A, p) d B_{1}(b) .
$$


Notice that constrained efficiency is a property of trading rules, and thus is independent of the choice of a price rule $p$. The "constraint" in constrained efficiency is embedded in the definition of trading rule, which respects the matching process.

Constrained efficiency is the same as constrained Pareto efficiency if ex-ante transfers of the divisible good can be made among the buyers, and among the sellers. Without such transfers, constrained efficiency as we have defined it is utilitarian and thus may rule out some constrained Pareto efficient allocations. To see the difference, consider an example where some sellers are forced to trade with any buyer that they meet in the first period whose valuation falls below a certain level, even if the buyer's value is less than the seller's. Such trades can be part of a constrained Pareto efficient allocation if no transfers are permitted, since these sellers take low valued buyers out of the market, which leads to higher expected utilities for the other sellers because the remaining pool of buyers has a higher average valuation. This sort of trading fails our definition of constrained efficient allocation since it does not maximize the overall gains from trade.

Our definition of efficiency takes the set of agents in the system as given. If one allowed control of the set of agents present, then a perfectly informed planner could induce the extra-marginal traders to leave, by mandating that all trade be consummated in the first period, at the competitive price. We rule out such a scheme by taking the agents present in the initial matching process as exogenous. Moreover, any of a number of embellishments of the model would nullify schemes of this sort. For example, if there is some aggregate uncertainty about the distribution of buyers or sellers (e.g., a finite number of traders sampled from a known distribution), then the competitive price is not known with certainty and every trader could have some probability of being on the right side of the market clearing price. ${ }^{15}$ Alternatively (as in Shimer and Smith (1994)), if there is some match-specific component of the valuations, so that the value we model is only the expectation of a value which may vary with the match (or just over time), then even traders who have a low expected gain from trade may still have a significant option value and an incentive to stay in the market. Finally, admitting convex preferences and divisibilities (as in a classical Edgeworth box) would offer potential gains from trade to almost all agents even though some could be very small. Rather than complicate the model in one of these ways we simply take the matching process to be exogenous.

\section{CHARACTERIZATION OF CONSTRAINED EFFICIENCY}

Our analysis of constrained efficiency is restricted to the case of $T=2$. (See Jackson and Palfrey (1997) for some results on arbitrary finite horizons.) We begin with an illustrating example and then turn to the characterization result.

${ }^{15}$ Our use of the continuum as a simplifying tool is responsible for the departure from this. 
EXAMPLE 1: Consider a case where $\delta=1$, buyers' valuations are uniformly distributed across $[0,1]$ with a total mass of 1 , and a mass $0<m<1$ of sellers have valuation 0 and the remaining mass, $1-m$, have valuation 1 . This is represented by $B_{1}(b)=b$ for all $b$ and $S_{1}(s)=m$ for all $s<1$.

In the absence of matching considerations or any frictions, Pareto efficient allocations would involve the assets going to the buyers with value at least $1-m$. The competitive allocations are an obvious choice, where sellers sell to the buyers with values above the competitive price, $p=1-m$. In our model, trade is constrained through the matching process, and the characterization of an efficient allocation becomes complicated since some of the higher value bùyers might never be matched to a seller with whom they can trade, and it is sometimes better to clear a trade with a low-valued buyer than to wait for a buyer with a higher expected value.

It is straightforward, but instructive, to derive the constrained efficient allocation rule for this example. In the second (last) period, all positive value trades should be cleared, since there will be no further matching. It is also clear that a constrained efficient trading rule will be a cutoff rule, so it suffices to specify the minimum value of a buyer that should trade in the first period if matched with a 0 value seller. (These and other claims in this example are proved in Theorem 1.) For any value $c$ set as a cutoff today, the remaining distribution tomorrow will be $B_{2}(b)=b$ for $b \leq c$, and $B_{2}(b)=(1-m)(b-c)+c$ for $b>c$. The gain from clearing a trade today with a buyer of value $b$, is simply $b$. Sellers who do not trade today are rematched in the second period. The expected value of the buyer with whom they will trade in the second period is simply the expected value of $b$ under the distribution $B_{2}(v) / B_{2}(1)$, which is

$$
\frac{1-m(1-c)(1+c)}{2(1-m(1-c))} \text {. }
$$

The constrained efficient trading rule is obtained by equating the cutoff value equal to the expected value of rematching. That is, on the margin, a trade should be cleared today if (and only if) it offers at least as much total value as could be expected by waiting and clearing the trade tomorrow. Solving for $c^{*}$, the efficient cutoff rule is

$$
c^{*}=\frac{\sqrt{1-m}-(1-m)}{m} .
$$

The cutoff rule is decreasing in $m$. As the mass of sellers $m$ increases, the current cutoff has less of a reduction effect on tomorrow's expected trading value. Also notice that the cutoff value is always lower than the competitive price $(1-m)$.

The efficient solution in the above example has an easily characterizable form since sellers are effectively homogeneous, but in many ways it is representative of the characterization which is provided below for the case of general distributions of buyer and seller valuations. 
THEOREM 1: There exists a unique (up to sets of measure 0 ) constrained efficient trading rule. It is described by cutoff rules, with associated functions $\beta_{1}(s)$ and $\beta_{2}(s)$. These cutoff rules uniquely satisfy the following equations.

$$
\beta_{2}(s)=s, \quad \forall s \in[0,1],
$$

and

$$
\begin{aligned}
\beta_{1}(s)-s= & \delta \int_{0}^{1} \max \left[b^{\prime}-s, 0\right] \frac{d B_{2}\left(b^{\prime}\right)}{B_{2}(1)}+\delta \int_{0}^{1} \max \left[\beta_{1}(s)-s^{\prime}, 0\right] \frac{d S_{2}\left(s^{\prime}\right)}{S_{2}(1)} \\
& -\delta \int_{0}^{1}\left(\int_{0}^{1} \max \left[b^{\prime}-s^{\prime}, 0\right] \frac{d B_{2}\left(b^{\prime}\right)}{B_{2}(1)}\right) \frac{d S_{2}\left(s^{\prime}\right)}{S_{2}(1)},
\end{aligned}
$$

if this is feasible with $\beta_{1}(s)<1$, and $\beta_{1}(s)=1$ otherwise, where $S_{2}$ and $B_{2}$ are determined by (1) and (2), respectively. Furthermore, $\beta_{1}(s)$ is continuous and is strictly increasing at values of s such that $\beta_{1}(s)<1$.

Let us examine the intuition behind (3) as a characterization of efficiency. Consider a planner deciding whether to clear a currently matched pair, with valuations $s$ and $\beta_{1}(s)$. Since $\beta_{1}(s)$ is the cutoff value for $s$, the planner should be indifferent between clearing this trade or not. If this trade is cleared, then the left-hand side represents the marginal value ${ }^{16}$ of consummating that trade today. If this trade is not cleared, then $s$ and $\beta_{1}(s)$ will be put back in the pool in the second period. The right-hand side gives the marginal expected value from throwing both players back in the pool to be rematched tomorrow. This marginal expected value has three components. Throwing the players back means that they are matched with two other players who would have been otherwise matched. Given that those two other players will be randomly selected, on average one can treat the opportunity cost for matching them with $s$ and $\beta_{1}(s)$ as being the average trade value in the second period. This is the last expression in (3). The net value that comes from the random rematching of $s$ and $\beta_{1}(s)$ is then the expected value from each of their rematchings (the first two expressions on the right-hand side of (3)), less the opportunity cost of the agents with whom they are rematched (the last expression on the right-hand side of (3)).

\section{NECESSARY CONDITIONS FOR VOLUNTARY IMPLEMENTATION} AND ATTAINABILITY

Next, we turn to the issue of voluntary implementation and consider the case of arbitrary (finite) $T$. Characterizations of voluntary implementation and attainability provide us with the complete collection of allocation rules that could ever

\footnotetext{
${ }^{16}$ Each trade is in fact of measure 0 , so a calculus of variations argument is used in the formal derivation.
} 
be the equilibrium outcomes of such a dynamic interaction - under any negotiation process (which is representable by a finite extensive game form of perfect information). With such characterizations in hand, we will return to check whether constrained efficient allocations are attainable.

\section{Negotiation and Game Forms}

The formal, or informal, negotiation process that goes on between a buyer and seller who are matched at time $t$ is represented by an extensive game form $\gamma_{t}$. This game form is the same across all pairs matched at time $t$. The game form $\gamma_{t}$ is a finite stage extensive game form of perfect information. (The results extend to infinite stage game forms, but finite ones are all that are needed.)

Since $\gamma_{t}$ can depend on time, in equilibrium it can also depend on the measures of agents remaining. However, $\gamma_{t}$ cannot depend on the history of play. This is essentially an anonymity restriction so that the mechanism cannot respond to the particular actions of any agent, which is motivated by our interest in modeling ; markets. If one permits the mechanism to depend fully ${ }^{17}$ on the history, the implementation problem can become trivial. The future stages of the mechanism could then be chosen to enforce no trade if any agent deviates from prespecified actions. This defeats the idea of individual rationality as capturing voluntary trade with an endogenous outside option, as the outside option 'could be controlled as a function of any single agent's actions. If that were the case, the mechanism would then simply reduce to a forcing contract.

One can argue that we should use the stronger assumption that the mechanism be the same in each period. That is, the form of negotiation available at any time should be the same if it is representing some primitive set of available actions. While we agree with this in certain contexts (for instance in a richer model where there are balancing inflows of agents too), allowing for the larger set of mechanisms strengthens our impossibility result, and is congruent with the fact that the stock of agents in our model is nonstationary. It is possible that the bargaining procedure could depend on market conditions (for instance, by convention who makes the first offer in an alternating bargaining procedure might depend on the relative excess supply or demand). Of course, a stationary mechanism is a special case of the ones we consider here, and our characterization of implementation can be specialized to that case.

\section{The Signature Stage}

The heart of our analysis is the assumption that no agreement becomes binding until it is signed by each of the two agents. After negotiations have led to a suggested trade and price, the trade does not take place unless both agents "sign" the agreement. This is captured as follows. Consider, $\gamma_{t}$, an extensive game form with perfect recall to be played between an arbitrary buyer and seller

${ }^{17}$ We could allow $\gamma_{t}$ to depend on the history of play up to sets of measure 0 . 
at some time $t$, such that each terminal node suggests either a trade and price, or no trade. Given $\gamma_{t}$, let us define a dynamic version, $\Gamma\left(\gamma_{t}\right)$, as follows. First, replace any terminal node of $\gamma_{t}$ which recommends a trade and price, with a node that has a binary choice node (yes, no) for the buyer. Let "no" lead to a terminal node with no trade as the outcome. Let "yes" lead to a binary choice node (Yes, No) for the seller. Let "No" lead to a terminal node with no trade as the outcome, and "Yes" lead to a terminal node with the originally prescribed trade and price. We have simply augmented $\gamma_{t}$ by additional moves that require both the buyer and seller's "signature" before completing the trade.

At any time $t$, each matched buyer and seller play the augmented version of $\gamma_{t}$. If the outcome of $\Gamma\left(\gamma_{t}\right)$ is trade, then the trade is consumated and the buyer and seller are removed from the matching process. If the outcome is no trade, then the buyer and seller are returned to their respective pools to be rematched in the next period.

As an example, consider a simple dictatorial mechanism $\gamma_{t}$ where the seller simply announces a price $p \in[0,1]$ and the outcome is then trade at price $p$. The augmented version $\Gamma\left(\gamma_{t}\right)$ has the seller announce $p \in[0,1]$ as the first stage. Next, the buyer, having observed $p$, chooses from (yes, no). Finally, the seller, having observed $p$ and the buyer's move, chooses from (Yes, No). The outcome of $\Gamma\left(\gamma_{t}\right)$ is trade at price $p$ if the choices in the "signature" stages are yes and Yes; and no trade (return for rematching at time $t+1$ ), otherwise.

\section{Equilibrium}

A buyer's strategy for time $t$ is a measurable function, $\sigma_{t}^{b}(s, b)$, mapping pairs of buyer and seller valuations into the set of behavioral strategies for the buyer role in $\Gamma\left(\gamma_{t}\right)$. A seller's strategy for time $t, \sigma_{t}^{s}(s, b)$, is similarly defined. A collection of pure ${ }^{18}$ strategies $\sigma=\left(\sigma_{1}^{b}, \ldots, \sigma_{T}^{b} ; \sigma_{1}^{s}, \ldots, \sigma_{T}^{s}\right)$ induces an allocation rule $\left(A_{\sigma}, p_{\sigma}\right)$.

An equilibrium of the augmented sequence of mechanisms is a specification of strategies $\sigma$ such that for each $t$ and $(s, b)$ :

(i) $\sigma_{t}^{b}(s, b)$ and $\sigma_{t}^{s}(s, b)$ form a subgame perfect equilibrium of $\Gamma\left(\gamma_{t}\right)$, where the utility of no-trade is evaluated as $\delta u_{t+1}^{b}\left(b, A_{\sigma}, p_{\sigma}\right)$ for buyers and $\delta u_{t+1}^{s}\left(s, A_{\sigma}, p_{\sigma}\right)$ for sellers $(0$ if $t=T),{ }^{19}$ and

(ii) at any node where an agent's actions may lead either to current trade at some price or to rematching, the agent chooses an action leading to rematching only if it offers an expected utility higher than any of the other available actions.

\footnotetext{
${ }^{18}$ In this model, mixed strategies will not play a role in any equilibrium.

${ }^{19}$ This definition is stronger than simply defining an equilibrium to be a subgame perfect equilibrium of the overall game form with the continuum of players and $T$ periods. The overall game form has many interlaced information sets (as agents do not know the play of all the other agents in preceding periods) and so it does not have proper subgames-so subgame perfection applied overall would simply boil down to Nash equilibrium. The definition of equilibrium we employ applies subgame perfection directly to each time and match and thus avoids such a problem.
} 
Part (i) of the definition of equilibrium imposes sequential rationality in the form of subgame perfect equilibrium. Part (ii) of the definition of equilibrium is a tie-breaking rule when an agent is indifferent between trading today or waiting and being rematched. The particular form of the tie-breaking rule is not important: we could have defined it to have agents always favoring delay in such situations. One can think of this as being equivalent to a lexicographic preference assumption that eliminates indifference. ${ }^{20}$ This simplifies the analysis, as it produces a unique prediction of an outcome of a given extensive game form as a function of endogenous reservation prices (although there can still exist multiple equilibria because of the endogeneity as in Example 2).

\section{Voluntary Attainability and Implementability}

An allocation rule $(A, p)$ is voluntarily attainable if there exist $\left(\gamma_{1}, \ldots, \gamma_{T}\right)$ such that at least one equilibrium of the augmented sequence of mechanisms results in an allocation rule that is equivalent to $(A, p)$.

The difference between attainability and implementability is uniqueness. Attainability does not require uniqueness, and hence is a very weak form of implementation. ${ }^{21}$ More generally, one may be interested in knowing all the equilibria of a mechanism, which motivates the definition below.

An allocation rule $(A, p)$ is voluntarily implementable if there exist $\left(\gamma_{1}, \ldots, \gamma_{T}\right)$ such that each equilibrium of the augmented sequence of mechanisms results in an allocation rule that is equivalent to $(A, p)$.

Alternatively, we may simply be concerned that an efficient trading rule be implemented (or attainable) and not concerned with the particular prices that are realized. We say that a trading rule $A$ is voluntarily implementable if there exists a sequence $\gamma_{t}$ such that for each equilibrium there exists a price rule $p$ such that the equilibrium results in an allocation rule equivalent to $(A, p)$. A trading rule $A$ is voluntarily attainable if there exists a sequence $\gamma_{t}$ such that there exists some equilibrium and price rule $p$ such that the equilibrium results in an allocation rule equivalent to $(A, p)$.

Rubinstein and Wolinsky (1992) provide a mechanism for subgame perfect implementation in a pairwise bargaining model, but in their model there is no possibility of rematching. Thus, in their model the agents' reservation values are fixed. Given the possibility of rematching we consider here, the reservation values of the agents become endogenous to the equilibrium. This provides serious complications to the implementation problem. We end up having a necessary condition of nondecreasing prices which is similar to Rubinstein and Wolinsky's, except that it is stated relative to the endogenous valuations. Also,

\footnotetext{
${ }^{20}$ We did not model it that way since it would preclude a utility representation.

${ }^{21}$ This is roughly equivalent to what has been known in the literature as "truthful" implementation in the case where implementation is possible in direct mechanisms (Dasgupta, Hammond, and Maskin (1979)). We do not consider direct mechanisms given the dynamic and voluntary nature of the problem, so we have defined attainability.
} 
we end up with a strong version of an additional individual rationality condition that relates the entire set of prescribed prices (thus the prices available through the mechanism) to the endogenous reservation values.

First, let us examine the condition which is the appropriate generalization of the Rubinstein and Wolinsky (1992) condition to say that the prices be nondecreasing in the endogenous reservation values. This distinction between valuations and endogenous reservation values is very important since reservation values are not always nondecreasing in an agent's primitive valuation, as reservation prices depend on future prospects for trade under an allocation rule.

Nondecreasing Prices: An allocation rule $(A, p)$ has nondecreasing prices as a function of reservation prices, if for each $t,(s, b)$, and $\left(s^{\prime}, b^{\prime}\right)$ in $A_{t}$ :

$$
p_{t}(s, b) \geq p_{t}\left(s^{\prime}, b^{\prime}\right)
$$

whenever $\bar{p}_{t}^{s}(s ; A, p) \geq \bar{p}_{t}^{s}\left(s^{\prime} ; A, p\right)$ and $\bar{p}_{t}^{b}(b ; A, p) \geq \bar{p}_{t}^{b}\left(b^{\prime} ; A, p\right)$.

Notice that an implication of the above condition is that the price rule can only vary with the reservation prices of the agents.

The necessity of nondecreasing prices is verified as follows. Since the bargaining game has a finite extensive form with perfect information and agents have strict preferences over outcomes, the equilibrium outcome for every $(s, b)$ (fixing reservation prices) is unique. Let $p=p(s, b)$ be the outcome for the pair $(s, b)$, and we consider $b^{\prime}$ with a higher reservation value $\bar{p}_{t}^{b^{\prime}}>\bar{p}_{t}^{b}$. Since only the buyer's valuation has changed, either $p$ is still an equilibrium outcome, or there is a new equilibrium and the $b^{\prime}$ buyer must have at least one strictly improving deviation somewhere in the game tree. Given the change in preferences of the buyer, the only way a deviation can be improving (and not have been improving before) is for the deviation to lead to a price between $\bar{p}_{t}^{b^{\prime}}$ and $\bar{p}_{t}^{b}$, while the previous outcome was no trade. For this to have an effect further up the tree, it must be that an agent chooses this price rather than another one or no trade further up the tree, and so the change in the higher subgame must result in this price. This logic is iterated back to the equilibrium path, which implies that changes can only result in a higher price. Increasing the seller's reservation value has similar implications.

In addition to the nondecreasing price condition, an additional condition will be necessary. Given the individual rationality that is at the heart of our definition of voluntary implementation, it is clear that the trades suggested under an implementable (or attainable) allocation rule must be individually rational: the price $p$ of any trade consummated between $s$ and $b$ in period $t$ must lie between the corresponding seller and buyer reservation values.

Individual Rationality: An allocation rule $(A, p)$ satisfies individual rationality if for any $t$ and $(s, b) \in A_{t}$

$$
\bar{p}_{t}^{s}(s ; A, p) \leq p_{t}(s, b) \leq \bar{p}_{t}^{b}(b ; A, p) .
$$


Although it is obvious that individual rationality is necessary for voluntary attainability, it is more subtle that a stronger condition is necessary for voluntary attainability. This stronger version of individual rationality states that there is no price at which some pair of agents trade at time $t$ that is simultaneously individually rational for some other pair of agents who should not trade under the allocation rule.

Strong Individual Rationality: An allocation rule $(A, p)$ satisfies strong individual rationality if it satisfies individual rationality, and for each $t,\left(s^{\prime}, b^{\prime}\right) \notin A_{t}$, and $(s, b) \in A_{t}$, either

$$
p_{t}(s, b)>\bar{p}_{t}^{b}\left(b^{\prime} ; A, p\right)
$$

or

$$
p_{t}(s, b)<\bar{p}_{t}^{b}\left(s^{\prime} ; A, p\right)
$$

To understand the necessity of this condition suppose that there are agents who should not trade under the desired allocation rule, and there is a mutually individually rational price given their anticipated values from rematching, and this price is available at some terminal node in the tree. Tracing the path from this terminal node back up the tree, one can find a best response for each agent at each node and this must leave them at least as well off as trade at this price. In this way one can show that there exists an equilibrium that involves trade between these two agents. However, from the uniqueness of the equilibrium outcome for a given pair of agents in their round of bargaining (fixing their anticipated reservation prices under equilibrium rematchings), they must trade in every equilibrium, which would contradict attainability.

We can summarize the conditions that are necessary for voluntary attainability, and thus for voluntary implementability.

THEOREM 2: Consider a trading rule, $A$, that is voluntarily attainable (or implementable), and $(\hat{A}, \hat{p})$, an allocation rule corresponding to one of the equilibria of an implementing mechanism, where $\tilde{A}$ is equivalent to $A$. Then $(\hat{A}, \hat{p})$ satisfies strong individual rationality and has nondecreasing prices.

We make two remarks on Theorem 2. First, these conditions are necessary even when one just considers attainability. In other words, these conditions are needed simply to ensure that $(\hat{A}, \hat{p})$ can arise as an equilibrium of any mechanism. The conditions are not arising from multiple equilibrium considerations. Second, these conditions are still necessary for voluntary implementability when one admits infinite stage mechanisms. Details on this are given in a footnote to the proof (see Appendix).

The conditions of nondecreasing prices and strong individual rationality play a central role in the full characterizations of voluntary attainability and implementation. The full characterization tackles difficulties associated with possible 
discontinuities in the implemented price function, as well as the usual implementation challenge of ruling out equilibria which do not result in an allocation rule equivalent to $(A, p)$. However, in some cases of interest the conditions of nondecreasing prices and strong individual rationality are sufficient for voluntary attainability. Let us describe a mechanism that will show this.

Given a set of prices $P \subset[0,1]$, denote

$$
\operatorname{IR}(P)=\left\{(q, r) \in[0,1]^{2} \mid \exists p \in P, q \leq p \leq r\right\} .
$$

Fix an allocation rule $(A, p)$ and let $R_{t}$ be the range of $p_{t}$ over $A_{t}$. Under nondecreasing prices $p_{t}$ can be rewritten as a function of the endogenous reservation values $p_{t}\left(\bar{p}^{s}, \bar{p}^{b}\right)$. Consider the following mechanism: ${ }^{22}$

Stage 1: The seller announces $p^{s}$. Proceed to Stage 2.

Stage 2: The buyer announces $p^{s^{\prime}}, p^{b}$. Proceed to Stage 3.

Stage 3: The seller announces $p^{b^{\prime}}$.

The Outcome Function:

If $p^{b^{\prime}} \leq p^{b}$ and $p^{s^{\prime}} \geq p^{s}$ and $\left(p^{s^{\prime}}, p^{b^{\prime}}\right) \in I R\left(R_{t}\right)$, then the outcome is $p_{t}\left(p^{s^{\prime}}, p^{b^{\prime}}\right)$. If $p^{b^{\prime}} \leq p^{b}$ and $p^{s^{\prime}}<p^{s}$ and $\left(p^{s^{\prime}}, p^{b}\right) \in I R\left(R_{t}\right)$, then the outcome is inf $p \in$ $\left.R_{t} \mid p \geq p^{s^{\prime}}\right\}$.

If $p^{b^{\prime}}>p^{b}$ and $\left(p^{s}, p^{b^{\prime}}\right) \in I R\left(R_{t}\right)$, then the outcome is $\sup \left\{p \in R_{t} \mid p \leq p^{b^{\prime}}\right\}$.

Otherwise, the outcome is no trade.

Consider the case where $B_{1}$ and $S_{1}$ are continuous and increasing. Let $(A, p)$ be an allocation rule such that $A_{t}$ is a continuous cutoff rule, ${ }^{23}$ and $p_{t}$ is continuous on $A_{t}$ for all $t$.

Given these continuity and monotonicity conditions on the allocation rule and the buyer and seller distributions, nondecreasing prices and strong individual rationality are sufficient for voluntary attainability. One can easily verify that there is an equilibrium of the above mechanism where the announcement of $p^{s}$ and $p^{b}$ in equilibrium should be the true (endogenous) reservation prices of the agents. To see how this works, note that the announcement of $p^{s^{\prime}}$ allows the buyer to challenge the seller's announcement if, for instance, the seller announces $p^{s}>\bar{p}^{s}$. In that case, the buyer can revise the seller's announcement by saying $p^{s^{\prime}}<p^{s}$, thereby forcing the seller to either take that price or opt for no trade (where the seller will get her reservation value). If the seller honestly reveals $\bar{p}^{s}$, then a buyer has no incentive to challenge. Similar reasoning applies to the buyer's announcement of $p^{b}$ and the seller's possible challenge, $p^{b^{\prime}}$.

THEOREM 3: Suppose that $B_{1}$ and $S_{1}$ are continuous and increasing. Consider an allocation rule, $(A, p)$, such that $A_{t}$ is a continuous cutoff rule, and $p_{t}$ is continuous on $A_{t}$ for all $t .(A, p)$ is voluntarily attainable if and only if it satisfies strong individual rationality and has nondecreasing prices.

\footnotetext{
${ }^{22}$ Although the conditions needed for implementation in our setting turn out to be different, the mechanism used for implementation (or attaining an outcome) shares some features with the mechanism in Rubinstein and Wolinsky (1992).

${ }^{23}$ That is, the cutoff values from both the buyer and seller's perspectives are continuous functions.
} 


\section{A Characterization of Voluntary Implementation}

Theorem 3 characterized voluntary attainability for situations where the distributions and allocation rule are well behaved. We now consider general distributions and allocation rules, and deal explicitly with the multiple equilibrium problem that is inherent in the endogeneity of reservation prices and thus voluntary implementation.

First, we extend the necessary conditions for the case of general distributions and allocation rules.

$\because$ An allocation rule $(A, p)$ satisfies voluntary trade if for each $t$ there exists $P_{t} \subset[0,1]$ and $\hat{p}_{t}:[0,1]^{2} \rightarrow P_{t}$ such that:

(V1) [Reservation Price Measurability] For every $s, b \in A_{t}, p_{t}(s, b)=$ $\hat{p}_{t}\left(\bar{p}_{t}^{s}(s), \bar{p}_{t}^{b}(b)\right)$.

(V2) [Individual Rationality] $\left(\bar{p}_{t}^{s}(s), \bar{p}_{t}^{b}(b)\right) \in I R\left(P_{t}\right)$, for every $s, b \in A_{t}$, and $\bar{p}^{s} \leq \hat{p}_{t}\left(\bar{p}^{s}, \bar{p}^{b}\right) \leq \bar{p}^{b}$, for every $\left(\bar{p}^{s}, \bar{p}^{b}\right) \in \operatorname{IR}\left(P_{t}\right)$.

(V3) [Strong Individual Rationality] $\left(\bar{p}_{t}^{s}(s), \bar{p}_{t}^{b}(b)\right) \notin I R\left(P_{t}\right)$, for every $s, b \notin$ $A_{t}$.

(V4) [Nondecreasing Prices] $\hat{p}_{t}$ is nondecreasing over the domain $\operatorname{IR}\left(P_{t}\right)$.

(V5) [Separating Prices] for every $\left(\bar{p}^{s^{\prime}}, \bar{p}^{b}\right) \in I R\left(P_{t}\right)$ and $\bar{p}^{s}$ such that $\left(\bar{p}^{s}, \bar{p}^{b}\right)$ $\in \operatorname{IR}\left(P_{t}\right)$, if $\hat{p}_{t}\left(\bar{p}^{s^{\prime}}, \hat{p}^{b}\right)<\hat{p}_{t}\left(\bar{p}^{s}, \bar{p}^{b}\right)$, then there exists $p \in P_{t}$ such that $\bar{p}^{s^{\prime}} \leq p<$ $\bar{p}^{s}$. Similarly, for every $\bar{p}^{b^{\prime}}$ if $\hat{p}_{t}\left(\bar{p}^{s}, \bar{p}^{b^{\prime}}\right)>\hat{p}_{t}\left(\bar{p}^{s}, \bar{p}^{b}\right)$, then there exists $p \in P_{t}$ such that $\bar{p}^{b^{\prime}} \geq p>\bar{p}^{b}$.

Let us discuss some of the differences in the above condition from the conditions stated previously. The conditions (V2)-(V4) are direct extensions of the corresponding previous conditions. The set $P_{t}$ corresponds to the set of prices that are reachable by the implementing mechanism. Sometimes it is necessary for this to be larger than the set of prices which are supposed to be traded at in equilibrium, as off equilibrium behavior will be important in determining equilibrium behavior (see Example 2, below). Then, for instance, the strong individual rationality condition must be satisfied relative to all of the prices in $P_{t}$. If some price in $P_{t}$ is individually rational, then an equilibrium which results in trade will exist. So (V3) must hold relative to all of $P_{t}$.

Condition (V1) is new relative to the nondecreasing prices and strong individual rationality. The function $\hat{p}_{t}$ has as its domain reservation prices, as these are what matter in determining equilibrium actions. It is necessary then that the implemented price function be measurable with respect to reservation prices, which is condition (V1).

The last condition (V5) is also added for the general case. It states that the implemented price function can only be increasing in places where we can distinguish the reservation prices of the agent in question. If, for instance, $\bar{p}^{s^{\prime}}<\bar{p}^{s}$, but there are no available prices from $P_{t}$ in between $\bar{p}^{s^{\prime}}$ and $\bar{p}^{s}$, then these two types would have exactly the same preferences over trades in $P_{t}$ (the only ones possible from the implementing mechanism). In such a case, the equilibrium actions of these two types must be the same. 
The voluntary trade condition is thus necessary both for voluntary attainability and voluntary implementability. However, voluntary implementability requires an additional necessary condition to avoid multiple equilibria, as illustrated in the following example.

EXAMPLE 2: Consider the constrained efficient trading rule defined in Example 1 , when $m=1 / 2$.

Consider a fixed price of $c=\sqrt{2}-1$ in the first period, which corresponds to $c^{*}$. So 0 -valued sellers trade with all buyers with values above $\sqrt{2}-1$ in the first period at a price of $\sqrt{2}-1$. In the second period let 0 -valued sellers trade with all buyers, and trade at a price equal to the buyer's valuation.

The voluntary trade condition is satisfied relative to this $A, p$ by setting $P_{1}=$ $\{\sqrt{2}-1\}, P_{2}=[0,1], \hat{p}_{1}\left(\bar{p}^{s}, \bar{p}^{b}\right)=\sqrt{2}-1$, and $\hat{p}_{2}\left(\bar{p}^{s}, \bar{p}^{b}\right)=\bar{p}^{b}$. It is then simple to verify (i)-(v). There exists a mechanism that has $A, p$ as an equilibrium: In the first period trade is simply at price $\sqrt{2}-1$. In the second period the seller makes take-it-or-leave-it offers to the buyer (and the seller can name any price in $[0,1])$.

First, we check that there is an equilibrium that results in $(A, p)$. It is the obvious one. Buyers approve trade in the first period if and only if $b \geq \sqrt{2}-1$, and the 0 -valued sellers approve trade in the first period. Notice that from the characterization of constrained efficiency (and from Example 1), we know that a 0 -valued seller's reservation price is exactly $\sqrt{2}-1$ in the first period. In the second period, 0 -valued sellers make the offer of $b$ to the buyer with which they are matched, and it is approved.

But there is another equilibrium relative to the above mechanism! It involves all of the sellers rejecting the first period price. The second period is as before. This is an equilibrium, since if all the sellers reject in the first period, then the full mass of buyers is still there in the second period. The average value of the buyers is then $1 / 2$ in the second period. Since this is larger than $\sqrt{2}-1$ (see Example 1), the sellers are indeed acting optimally. Since there are two equilibria, this does not implement the efficient solution. ${ }^{24}$

Nonetheless, the efficient allocation rule can be fully implemented by an alternative mechanism which is a simple variation on the above mechanism. Consider the following change: In the first period the buyer makes a take-it-orleave-it offer to the seller from the set of prices $[\sqrt{2}-1,1]$. Any buyer with à value above $\sqrt{2}-1$ would rather trade in the first period, since they expect to have their full value extracted in the second period. High valued buyers can offer sellers enough to get them to trade in the first period, even if the sellers expect a value above $c$ in the second period. This means that the trades will occur in the first period that should. Given that they occur, the buyers will be able to offer $\sqrt{2}-1$ and get it.

\footnotetext{
${ }^{24}$ In fact, the efficient equilibrium to the above mechanism is fragile: even a small variation in the expectations makes it better for the sellers to wait.
} 
The mechanism works because it has a range of available prices in the first period that is larger than just $\sqrt{2}-1$. This illustrates the important role of $P_{t}$ in the voluntary trade condition. It also gives us insight to the full characterization of implementation and the relationship to attainability: it must be that $(A, p)$ is attainable, but other (nonequivalent) $(\tilde{A}, \tilde{p})$ 's are not attainable. If we set $P_{1}=$ $[\sqrt{2}-1,1]$, then the voluntary trade condition is not satisfied relative to the undesired allocation rule where all of the agents wait until the second period to trade, and so that allocation rule will not be an equilibrium outcome. In particular, (V3), strong individual rationality, is violated in this example relative to this $P_{t}$.

For the characterization of implementation, we restrict attention to mechanisms that have the property that there exists a subgame perfect equilibrium of the augmented mechanism for each $t$ relative to every set of reservation prices $\bar{p}^{s}, \bar{p}^{b}$. We call these mechanisms closed. This avoids the use of controversial implementation theory "tricks" which exploit nonexistence of best responses in some portion of the message space.

THEOREM 4: If an allocation rule $(A, p)$ is voluntarily implementable by a closed mechanism, then:

(i) there exists $(\tilde{A}, \tilde{p})$ which is equivalent to $(A, p)$ and satisfies the voluntary trade condition, and

(ii) for each $\left(A^{\prime}, p^{\prime}\right)$ not equivalent to $(A, p),\left(A^{\prime}, p^{\prime}\right)$ fails to satisfy the voluntary trade condition relative to the same $\hat{p}$ and $P_{t}$ as $(\tilde{A}, \tilde{p})$.

Conversely, if (i) and (ii) hold and $P_{t}$ is closed for each $t$, then $(A, p)$ is voluntarily implementable by a closed mechanism.

We remark that (i) is necessary for voluntary attainability as well as implementability. This is proven in the Appendix.

Condition (i) states the necessity of voluntary trade, which we have discussed earlier. Condition (ii) is the condition ruling out undesired multiple equilibria, as illustrated in Example 2. The implementing mechanism used to prove sufficiency is a simple variation on the one described in the previous section, prior to Theorem 3 .

We know that it is not necessary that $P_{t}$ be closed. It is an open question whether (i) and (ii) are sufficient in the absence of this condition, or whether there are additional necessary conditions.

\section{ATTAINING OR IMPLEMENTING CONSTRAINED EFFICIENT RULES}

Given the characterizations of attainability and implementation, we turn to the issue of attaining or implementing efficient trading rules. Let us begin with an example that illustrates that the consideration of all bargaining procedures is important. There are efficient allocation rules that are not voluntarily attainable when one considers a procedure that results in Nash bargaining solutions, but 
are voluntarily attainable (and implementable) via alternative bargaining procedures.

EXAMPLE 3: This is a variation on Example 1, where there is discounting $(\delta<1)$ and where $m=\frac{1}{2}$. Again, buyers' valuations are uniformly distributed across $[0,1]$ with a total mass of 1 . A mass $\frac{1}{2}$ of sellers have valuation 0 and the remaining mass have valuation 1 . This is represented by $B_{1}(b)=b$ for all $b$ and $S_{1}(s)=\frac{1}{2}$ for all $s<1$.

Using Theorem 1 one can compute the cutoff value $c^{*}$ (i.e., $\left.c^{*}=\beta_{1}(0)\right)$ as the unique solution to the equation

$$
c^{*}=\delta\left(\frac{2-c^{*}}{2-\delta c^{*}}\right) E_{2}[b],
$$

which is the unique root in $[0,1]$ to the cubic equation

$$
\left(c^{*}+c^{* 2}\right)\left(2-\delta c^{*}\right)=\frac{\delta}{2}\left(1+c^{* 2}\right)\left(2-c^{*}\right) .
$$

Notice that if prices are set by the Nash bargaining solution so that $p_{t}(0, b)=$ $b / 2$, then a seller with $s=0$ will choose to trade with any $b \geq c$ where $c$ satisfies $c / 2=\delta E_{2}[b / 2]$, or

$$
c=\delta E_{2}[b]
$$

This is inefficient, since sellers fail to consummate all efficient trades in the first period. Thus, if one restricts attention to prices corresponding to Nash bargaining, then the efficient trading rule cannot be voluntarily attained. The source of the adverse incentives under the Nash bargaining price rule is that it splits the buyer and seller surplus in half. As a result, a seller matched with a buyer whose valuation is close to the efficient $c^{*}$ would prefer to wait because the trading prospects are more attractive tomorrow.

However, the efficient trading rule can be voluntarily attained when one considers a pricing rule that reduces the sellers' prospects tomorrow. A very simple modification of the Nash bargaining price rule accomplishes this: place a price ceiling on the transaction, equal to some value $P<1$. This changes the pricing rule from $p_{t}(0, b)=b / 2$ to $p_{t}(0, b)=\min [P, b / 2]$. For $\delta$ close to 1 there will exist a ceiling $P$ which creates the right incentives, where sellers matched with a buyer of valuation $c$ will be exactly indifferent between trading and waiting. To see this, note first that the right incentives will be provided as long as $P$ is chosen so that $P>\left(c^{*} / 2\right)$ and $E_{2}\left[p_{2}(0, b)\right]=\left(\left(2-c^{*}\right) /(2-\right.$ $\left.\left.\delta c^{*}\right)\right) E_{2}[b / 2]$. This will guarantee that the ceiling is not binding in the first period and that the cutoff value is chosen optimally. It is easy to see that $P$ can be chosen to accomplish this: $E_{2}\left[p_{2}(0, b)\right]$ varies continuously in $P$, ranging from 0 to $E_{2}[b / 2]$ so, for any value of $c \in[0,1]$, we can choose $P$ so that $E_{2}\left[p_{2}(0, b)\right]=((2-c) /(2-\delta c)) E_{2}[b / 2]$. When $\delta=1$ the solution for the opti- 
mum is $c^{*}=\sqrt{2}-1$ and the choice of $P=.5>\sqrt{2}-1$ works, and so for $\delta$ close to 1 the appropriate value of $P$ will satisfy $P>c^{*} / 2$, as required. This stationary pricing rule, which is a simple modification of Nash bargaining, offers exactly the right incentives to satisfy strong individual rationality, and together with the efficient trading rule is voluntarily attainable (and in fact implementable).

Example 3 shows that the efficient trading rule may not be attainable with a pricing rule determined by Nash bargaining, but could be attainable in conjunction with some other natural pricing rule (here Nash bargaining with a price cap). This illustrates why it is important to consider general bargaining procedures and general pricing rules in these matching/exchange environments. Consideration of only a single pricing rule, such as Nash bargaining, can significantly constrain the set of attainable or implementable allocations.

The Proposition below, however, shows that even admitting general bargaining procedures and pricing rules does not allow one to attain efficient allocation rules in some situations. Generally, there is a rich set of constraints imposed by strong individual rationality, and these can be difficult to satisfy when the distribution of sellers is more general than the one in the examples above.

PROPOSITION 1: There exists a robust set of continuous and increasing distributions of buyer and seller valuations for which the constrained efficient trading rule is not voluntarily attainable (and hence not voluntarily implementable).

The robustness mentioned in the Proposition refers to the fact that the result is true for any distributions satisfying the following ${ }^{25}$ for small enough $0<\epsilon<$ $1 / 2$ :

$$
\frac{1}{2}-\epsilon \leq B_{1}(\epsilon) \leq \frac{1}{2} \quad \text { and } \quad B_{1}(1-\epsilon) \leq \frac{1}{2}+\epsilon
$$

and likewise

$$
\frac{1}{2}-\epsilon \leq S_{1}(\epsilon) \leq \frac{1}{2} \quad \text { and } \quad S_{1}(1-\epsilon) \leq \frac{1}{2}+\epsilon .
$$

In other words, for small $\epsilon$ these distributions have nearly half their mass on values close to 1 and nearly half their mass on values close to 0 .

A sketch of the proof (details of which are found in the Appendix) is as follows: For such distributions, an efficient solution will clear trades in their first period between low valued sellers and high valued buyers and so the resulting distributions the second period will have approximately $1 / 3$ high value buyers and $2 / 3$ low valued buyers, and similarly $1 / 3$ low value sellers and $2 / 3$ high valued sellers. Thus, low valued sellers and high valued buyers (the only agents really generating gains from trade) have a chance of only $1 / 3$ of meeting a successful match in the second period. Since one side can get no more than half of the surplus of a successful match in the second period, either the low valued seller or the high valued buyer has an expected value of no more than $1 / 6$ from trading tomorrow. Say it is the low valued seller. The combination of individual

\footnotetext{
${ }^{25}$ In fact, the only nontrivial example of which we know where the constrained efficient allocation is voluntarily attainable is in the case of homogenous sellers.
} 
rationality and strong individual rationality imply that individual rationality is almost exactly binding for both the lowest valued seller (0) and her cutoff match $\left(\beta_{1}(0)\right)$, which in turn implies that the low valued seller can get no more than $1 / 6$ from her cutoff trade today. Since her cutoff match $\beta_{1}(0)$ is approximately $1 / 3$ (as derived from Theorem 1), the buyer with value $1 / 3$ must get at least $1 / 6$ from the trade today. However this buyer $\beta_{1}(0)$ can expect at most $(1 / 3) \times(1 / 3)=1 / 9$ from waiting and thus strictly prefers to trade today, which contradicts the fact that the individual rationality constraint should be binding at $\beta_{1}(0)$. The robustness follows from the fact that the efficient solution varies continuously with the distribution, so we can work with any distribution satisfying the above conditions for small $\epsilon$.

The rough intuition is that first period trades create an externality on the distribution of traders who are rematched in the next period. Thus in the optimal solution, it is possible that some "good" trading pairs ${ }^{26}$ (in the example a low valued seller and a low-middle valued buyer) should not trade and instead be left in the market to offset this externality. This can be true even though the expected surplus from that transaction in the first period exceeds the sum of the expected surpluses of the two transacting parties were they to search one more period. For any game that tries to implement this efficient solution, some of these trading pairs would prefer to trade in the first period, which prevents the efficient solution from being an equilibrium outcome.

\section{CONCLUDING REMARKS}

There are three main contributions in this paper.

First, we provide a characterization of constrained efficiency in a setting with random matching and search. In situations where markets are truly decentralized, standard notions of efficiency are inappropriate since goods may not be transferable arbitrarily from one agent to another. The matching process imposes constraints on the set of feasible allocations, and introduces search externalities across agents. These constraints and externalities are at the heart of the characterization of constrained efficiency.

Second, we provide characterizations of attainability and implementation in situations where mechanisms cannot impose trade on agents. The characterization is intuitive in terms of the (strong) individual rationality conditions that naturally arise from the voluntary choice of agents either to accept the outcome of the mechanism, or to reject it and search for a new trading partner in the next period. The implementation is shown to be achievable by simple mecha-

\footnotetext{
${ }^{26}$ This is the flip side of examples in Sattinger (1995) and Shimer and Smith (1994), where a congestion externality leads to too few trades taking place and removing low value trades to reduce congestion can be an efficiency gain. Here we find that the opposite problem can also occur: social gains can come from having some agents with attractive valuations stay in the market, while they may be too impatient.
} 
nisms using alternating move games with perfect information, with a structure similar to standard bargaining games.

Third, we show that it is often the case that constrained efficient allocations are inconsistent with voluntary decentralized trade under any bargaining game. Even with atomless agents, the externalities cannot be overcome, regardless of the mechanism by which agents negotiate and trade. Thus, in spite of the fact that trading pairs share complete information about each others' valuations, the strong necessary conditions imposed by voluntary trade are incompatible with overcoming the externalities and achieving efficient allocations.

The strength of the first two ${ }^{27}$ results we obtain is, of course, tempered by the fact that we have worked in a specific setting. The specific nature of the preferences of the agents (i.e., the "bargaining" structure), the way in which agents may accept or reject the suggestion of the mechanism, and the particular matching technology are important in terms of the clean and intuitive characterizations we obtain.

Relative to the implementation literature, this suggests exploring how the nonimposition restriction behaves in more general environments, especially those where one admits the possibility of some choices in matching, such as those offered by a centralized exchange. Relative to the competitive bargaining problem, it would be interesting to examine how the analysis extends to an infinite horizon, and to situations where there are inflows of agents.

In oùr introduction, we discussed our view that any negotiation and renegotiation should be modeled as part of the given game form. This viewpoint strengthens the conclusions of Proposition 1, since the result is true regardless of the form of negotiation that takes place. However, since we have not taken any stand on the particular process that may govern such interaction, our admissible class of game forms is still quite large. Although we impose restrictions of perfect information, finite length, signature stages, and lack of integer games, etc., we do not impose a priori restrictions on the specific structure of negotiation or renegotiation. So, if one considers an environment where there are natural or exogenously determined restrictions on how this process can take place, so that only some of the mechanisms that we have allowed are feasible, then additional conditions could come out of the characterization. We point out, however, that in spite of the larger class of mechanisms we have admitted, our theorems are proven without resorting to complicated or unnatural mechanisms. The implementing mechanisms used to prove the characterization results are extremely simple and involve only a sequential announcement of a reservation price by each agent, and an opportunity for the other agent to challenge this announcement with another price. Thus, in order for any a priori restrictions on negotiation (or renegotiation) to have an impact, they would have to rule out such mechanisms. Nevertheless, such mechanisms do allow for the imposition of no trade as an outcome even when there are mutual gains from trade to agents.

\footnotetext{
${ }^{27}$ The last result (the impossibility of implementing the efficiency rule) still holds in more general settings.
} 
Although this may be reasonable in some markets (e.g., security markets), it may not be in others (e.g., housing markets) where it will be necessary to rule out such mechanisms before one can take the sufficient conditions for implementation seriously.

Finally, in this model there are no transfers that are made except between the paired agents. Having a centralized authority that could execute transfers across agents and time could help avoid some of the negative externalities and help achieve efficiency. This is an important question for future investigation, and suggests interesting comparisons with centralized markets.

Division of Humanities and Social Sciences, 228-77, California Institute of Technology, Pasadena, CA 91125, U.S.A.; jacksonm@hss.caltech.edu; http: //www.hss.caltech.edu / jacksonm /Jackson.html

and

Division of Humanities and Social Sciences, 228-77, California Institute of Technology, Pasadena, CA 91125, U.S.A.; trp@hss.caltech.edu

Manuscript received November, 1996; final revision received October, 1997.

\section{APPENDIX}

Proof of THEOREM 1: First, any efficient trading rule must be (up to sets of measure 0 ) a cutoff rule for second period trades with $\beta_{2}(s)=s$, so $A_{2}=\{(s, b): b>s\}$. That is, almost every individually rational second period trade will be consummated.

Assuming this form for $\beta_{2}$, and using $\tilde{p}_{t}(s, b)=b$ (since $W$ is independent of $p$ ), we can write

$$
W\left(A_{1}\right)=\int_{0}^{1}\left(\int_{b \in A_{1}(s)}(b-s) d B_{1}(b)\right) d S_{1}(s)+\delta \int_{0}^{1} \int_{s}^{1}(b-s) \frac{d B_{2}(b)}{B_{2}(1)} d S_{2}(s)
$$

where $A_{1}(s)=\left\{b:(s, b) \in A_{1}\right\}$, and $B_{2}$ and $S_{2}$ are determined by (1) and (2).

Let us rewrite this as

$$
W(\pi)=\int_{0}^{1}\left(\int_{0}^{1} \pi(s, b)(b-s) d B_{1}(b)\right) d S_{1}(s)+\delta \int_{0}^{1} \int_{s}^{1}(b-s) \frac{d B_{2}(b)}{B_{2}(1)} d S_{2}(s),
$$

where $\pi(s, b)=1$ if $(s, b) \in A_{1}$ and $\pi(s, b)=0$ if $(s, b) \notin A_{1}$.

We maximize $W(\pi)$ with respect to all measurable $\pi$ 's and show that the unique solution corresponds to the claimed cutoff function in (3). Maximizing $W$ is a vector space optimization problem with the constraint $\pi(s, b) \in[0,1]$, for all $(s, b)$. A necessary condition for an optimum is that directional (Gâteaux) derivatives are either 0 or point inward from the boundary for almost all $(s, b)$. For our problem, this implies that, for almost all $(s, b), \pi=1$ when $(d[W(\pi)] / d[\pi(s, b)])>0$ and $\pi=0$ when $(d[W(\pi)] / d[\pi(s, b)])<0 .{ }^{28}$ Sufficiency of these conditions follows from the uniqueness of the solution, the continuity of $W$ and the compactness of the set of admissible $\pi(s, b)$ (in the weak $*$ topology).

${ }^{28}$ To be more explicit, the directional derivative with respect to some measurable function $h(s, b)$ in this case works out to be $\int_{(s, b)}(d[W(\pi)] / d[\pi(s, b)]) h(s, b) d(s, b)$. So, requiring that $\int_{(s, b)}(d[W(\pi)] / d[\pi(s, b)]) h(s, b) d(s, b) \leq 0$ for any $h$ such that $\pi(s, b)+h(s, b) \in[0,1]$ for all $(s, b)$, is equivalent to saying that for almost all $(s, b), \pi=1$ when $(d[W(\pi)] / d[\pi(s, b)])>0$ and $\pi=0$ when $(d[W(\pi)] / d[\pi(s, b)])<0$. 
Recall that

$$
S_{2}(v)=S_{1}(v)-\int_{s \leq v}\left(\int_{0}^{1} \pi(s, b) d B_{1}(b)\right) d S_{1}(s)
$$

and

$$
B_{2}(v)=B_{1}(v)-\int_{b \leq v}\left(\int_{0}^{1} \pi(s, b) d S_{1}(s)\right) d B_{1}(b) .
$$

Differentiate $W(\pi)$ with respect to $\pi(s, b)$ for any $(s, b)$, which leads to

$$
\begin{aligned}
\frac{d[W(\pi)]}{d[\pi(s, b)]}= & (b-s) d B_{1}(b) d S_{1}(s) \\
& +\frac{-\delta}{B_{2}(1)} \frac{d\left[B_{2}(1)\right]}{d[\pi(s, b)]} \int_{0}^{1}\left(\int_{s^{\prime}}^{1}\left(b^{\prime}-s^{\prime}\right) \frac{d B_{2}\left(b^{\prime}\right)}{B_{2}(1)}\right) d S_{2}\left(s^{\prime}\right)+ \\
& \delta \frac{d\left[d S_{2}(s)\right]}{d[\pi(s, b)]} \int_{s}^{1}\left(b^{\prime}-s\right) \frac{d B_{2}\left(b^{\prime}\right)}{B_{2}(1)}+\delta \frac{d\left[d B_{2}(b)\right]}{d[\pi(s, b)]} \int_{0}^{b}\left(b-s^{\prime}\right) \frac{d S_{2}\left(s^{\prime}\right)}{B_{2}(1)} .
\end{aligned}
$$

Next, observe that

$$
\begin{aligned}
& \frac{d\left[d S_{2}(s)\right]}{d[\pi(s, b)]}=-d B_{1}(b) d S_{1}(s), \\
& \frac{d\left[d B_{2}(b)\right]}{d[\pi(s, b)]}=-d B_{1}(b) d S_{1}(s),
\end{aligned}
$$

and

$$
\frac{d\left[B_{2}(1)\right]}{d[\pi(s, b)]}=-d B_{1}(b) d S_{1}(s) .
$$

Substituting these expressions into (A1) provides

$$
\begin{aligned}
\frac{d[W(\pi)]}{d[\pi(s, b)]}= & d B_{1}(b) d S_{1}(s)\left[(b-s)+\delta \int_{0}^{1}\left(\int_{s^{\prime}}^{1}\left(b^{\prime}-s^{\prime}\right) \frac{d B_{2}\left(b^{\prime}\right)}{B_{2}(1)}\right) \frac{d S_{2}\left(s^{\prime}\right)}{B_{2}(1)}\right. \\
& \left.-\delta \int_{s}^{1}\left(b^{\prime}-s\right) \frac{d B_{2}\left(b^{\prime}\right)}{B_{2}(1)}-\delta \int_{0}^{b}\left(b-s^{\prime}\right) \frac{d S_{2}\left(s^{\prime}\right)}{B_{2}(1)}\right] .
\end{aligned}
$$

Recall that we must have (almost everywhere) $\pi=1$ when $(d[W(\pi)] / d[\pi(s, b)])>0$ and $\pi=0$ when $(d[W(\pi)] / d[\pi(s, b)])<0$. To see that the solution should be a cutoff rule, fix $s$ and notice that the part inside the brackets on the right-hand side of the expression for $d[W(\pi)] / d[\pi(s, b)]$ is strictly increasing in $b$ since we assume $S_{1}(0)<S_{1}(1){ }^{29}$ Setting $(d[W(\pi)] / d[\pi(s, b)])=0$ implies (3). The continuity and increasing properties of $\beta_{1}(s)$ follow directly from inspection of the right-hand side of (3).

Q.E.D.

We next present the proofs of Theorems $2-4$. We do this in the order: Theorem 4, Theorem 2, Theorem 3. This is different from the order in the body of the paper, but it is the natural order to present the proofs, since the results in Theorem 4 are used to prove Theorems 2 and 3 . The claim in Section 6 and Proposition 1 are proved at the end.

${ }^{29}$ If $S_{1}(0)=S_{1}(1)$, then this derivative is constant in $b$ when $\delta$ equals 1 , and there are multiple solutions for the optimal first period allocation rule. 
ProOF OF THEOREM 4: We begin by demonstrating the necessity of the conditions. Suppose that ( $A, p)$ is implemented by $\left(\gamma_{1}, \ldots, \gamma_{T}\right)$ which satisfies the equilibrium existence condition stated prior to Theorem 4. Let $P_{t}$ be the set of prices that correspond to some terminal node of $\gamma_{t}$.

LEMMA: For any $t$, and for any $(s, b)$ pair, there is a unique subgame perfect equilibrium outcome of $\Gamma\left(\gamma_{t}\right)$ satisfying (ii) in the definition of equilibrium as a function of $\left(\bar{p}^{s}, \bar{p}^{b}\right)$. It is trade at some price if and only if $\left(\bar{p}^{s}, \bar{p}^{b}\right) \in I R\left(P_{t}\right)$.

Consider any $\left(\bar{p}^{s}, \bar{p}^{b}\right)$. By part (ii) of the definition of equilibrium, an agent's choice from a set of outcomes is uniquely determined. The subgame perfect equilibrium outcomes (which always exist under the existence condition on the mechanism) can thus be found by backward induction, which results in a unique outcome.

Only If: By the veto power that each agent has under the augmented mechanism, the unique equilibrium outcome must be no trade if $\left(\bar{p}^{s}, \bar{p}^{b}\right) \notin I R\left(P_{t}\right)$.

If: We now show that if $\left(\bar{p}^{s}, \bar{p}^{b}\right) \in \operatorname{IR}\left(P_{t}\right)$, then the unique equilibrium outcome must be trade at some price. Suppose the contrary, so that for some $\left(\bar{p}^{s}, \bar{p}^{b}\right) \in I R\left(P_{t}\right)$, the equilibrium outcome is no trade. Consider a pair of equilibrium strategies for $\left(\bar{p}^{s}, \bar{p}^{b}\right)$ when they are matched at time $t$ and denote these $\sigma$. These lead to no trade at time $t$. Consider also some strategies which lead to the outcome of $p$ at time $t$ and denote these $\sigma^{\prime}$. Alter $\sigma$ at each node on the play path of $\sigma^{\prime}$ to match the action under $\sigma^{\prime}$ at that node, and leave the actions at other nodes under $\sigma$ unchanged. Call this new strategy $\sigma^{\prime \prime}$. Since $\sigma^{\prime \prime}$ results in trade at $p$, it must not be an equilibrium for $\bar{p}^{s}$ and $\bar{p}^{b} .^{30}$ Find the last node along the play path of $\sigma^{\prime \prime}$ such that there is an improving deviation for the agent choosing at that node. Find a best response for that agent at that node. ${ }^{31}$ The new play path must lead to trade at some price since it is improving for that agent and both agents weakly prefer $p$ to no trade. The new strategy combination is now a Nash equilibrium in all subgames from this node on (and all subgames off the current play path). Iterate this logic up the nodes of the play path. This results in a subgame perfect equilibrium that has an outcome of trade at some price, which is a contradiction.

Q.E.D.

With the lemma in hand, we can conclude the proof of necessity in the theorem.

Define $\hat{p}_{t}$ to be the equilibrium price of $\Gamma\left(\gamma_{t}\right)$ as a function of $\left(\bar{p}^{s}, \bar{p}^{b}\right)$. By the lemma, this is a well defined function on the domain $\operatorname{IR}\left(P_{t}\right)$.

Let $(\tilde{A}, \tilde{p})$ denote an allocation rule corresponding to some equilibrium. By the definition of implementability, it is equivalent to $(A, p)$. Define $\bar{p}_{t}$ relative to the equilibrium strategies leading to $(\tilde{A}, \tilde{p})$.

We first verify (i). We show that voluntary trade holds relative to $(\tilde{A}, \tilde{p})$, for the $\hat{p}_{t}$ and $P_{t}$ defined above.

(V1) and (V3) follow directly from the lemma.

(V2) follows from the lemma and the fact that agents will never accept a price that is not individually rational in an equilibrium of the mechanism.

(V4): A sketch of the proof of this case was given in the text for increasing buyer's reservation values. The case of an increase in seller's reservation values is analagous, except the type of improving deviations is to have no-trade replace trade at some price. ${ }^{32}$

(V5): By (V4) we know that $\bar{p}^{s^{\prime}}<\bar{p}^{s}$. Suppose the contrary of (V5). Then for all $p \in P_{t}$, either $p \geq \bar{p}^{s}$ and $p \geq \bar{p}^{s^{\prime}}$, or $p<\bar{p}^{s}$ and $p<\bar{p}^{s^{\prime}}$. This implies that the set of equilibria is exactly the same for the two agents when either is matched with $\bar{p}^{b}$. This implies nonuniqueness of the equilibrium outcome, a contradiction.

\footnotetext{
${ }^{30}$ Notice that infinite stage mechanisms can be admitted and this proof still works, since $\sigma^{\prime}$ (and thus $\left.\sigma^{\prime \prime}\right)$ must result in trade after some finite number of stages.

${ }^{31}$ We know that there exists a best response at that node, since the other actions at that node yield the same outcomes that they would under $\sigma$, and there is a best response there under $\sigma$.

${ }^{32}$ Rubinstein and Wolinsky (1990) [Appendix II of the working paper version] offer a proof of a similar property.
} 
Next, let us verify (ii).

Consider an $\left(A^{\prime}, p^{\prime}\right)$ which is not equivalent to $(A, p)$. Consider the $\hat{p}_{t}$ and $P_{t}$ defined for each $t$ as above. Notice that (V4) and (V5) are satisfied, as they are independent of the allocation rule. We must show that one of (V1), (V2), and (V3) fail for $\left(A^{\prime}, p^{\prime}\right)$ relative to the $\hat{p}_{t}$ and $P_{t}$ defined above.

By the lemma, for each $t$ and $(s, b)$ there is a unique subgame perfect equilibrium outcome of $\Gamma\left(\gamma_{t}\right)$ relative to the reservation values $\left(\bar{p}_{t}^{s}\left(s ; A^{\prime}, p^{\prime}\right), \bar{p}_{t}^{b}\left(b ; A^{\prime}, p^{\prime}\right)\right)$. Select a subgame perfect equilibrium pair of strategies for each $t$ and $(s, b)$. By the implementation of $(A, p)$, these strategies cannot result in $\left(A^{\prime}, p^{\prime}\right)$. Thus, there exists $t$ and $(s, b)$ such that either:

Case 1: $(s, b) \notin A_{t}^{\prime}$ and the outcome is trade at some price $p$, or

Case 2: $(s, b) \in A_{t}^{\prime}$ and the outcome is trade at some price $p \neq p^{\prime}(s, b)$, or

Case 3: $(s, b) \in A_{t}^{\prime}$ and the outcome is no trade.

In Case 1, it follows from the lemma that $\left(\bar{p}_{t}^{s}\left(s ; A^{\prime}, p^{\prime}\right), \bar{p}_{t}^{b}\left(b ; A^{\prime}, p^{\prime}\right)\right) \in I R\left(P_{t}\right)$, which means that (V3) fails.

In Case 2, it follows from the definition of $\hat{p}_{t}$ that $p_{t}^{\prime}(s, b) \neq \hat{p}_{t}\left(\bar{p}_{t}^{s}\left(s ; A^{\prime}, p^{\prime}\right), \bar{p}_{t}^{b}\left(b ; A^{\prime}, p^{\prime}\right)\right)$, which means that (V1) fails.

In Case 3, it follows from the lemma that $\left(\bar{p}_{t}^{s}\left(s ; A^{\prime}, p^{\prime}\right), \bar{p}_{t}^{b}\left(b ; A^{\prime}, p^{\prime}\right)\right) \notin I R\left(P_{t}\right)$, which means that (V2) fails.

Sufficiency is established by constructing a mechanism that will implement any $(A, p)$ satisfying the voluntary trade condition. The mechanism $\gamma_{t}$ at time $t$ is the one described in Section 5:

Stage 1: The seller announces $p^{s}$. Proceed to Stage 2.

Stage 2: The buyer announces $p^{s^{\prime}}, p^{b}$. Proceed to Stage 3.

Stage 3: The seller announces $p^{b^{\prime}}$.

The Outcome Function:

If $p^{b^{\prime}} \leq p^{b}$ and $p^{s^{\prime}} \geq p^{s}$ and $\left(p^{s^{\prime}}, p^{b^{\prime}}\right) \in \operatorname{IR}\left(P_{t}\right)$, then the outcome is $\hat{p}_{t}\left(p^{s^{\prime}}, p^{b^{\prime}}\right)$.

If $p^{b^{\prime}} \leq p^{b}$ and $p^{s^{\prime}}<p^{s}$ and $\left(p^{s^{\prime}}, p^{b}\right) \in \operatorname{IR}\left(P_{t}\right)$, then the outcome is $\inf \left\{p \in P_{t} \mid p \geq p^{s^{\prime}}\right\}$.

If $p^{b^{\prime}}>p^{b}$ and $\left(p^{s}, p^{b^{\prime}}\right) \in \operatorname{IR}\left(P_{t}\right)$, then the outcome is $\sup \left\{p \in P_{t} \mid p \leq p^{b^{\prime}}\right\}$.

Otherwise, the outcome is no-trade.

We now prove sufficiency. Assume that (i) and (ii) hold. Consider the implementing mechanism, $\left(\gamma_{1}, \ldots, \gamma_{T}\right)$, described above. The remainder of the proof consists of verifying three claims.

ClaIM 1: Consider $t$ and a subgame perfect equilibrium of the augmented version of the mechanism described above under part (ii) of the definition of equilibrium, when reservation values are $\left(\bar{p}^{s}, \bar{p}^{b}\right)$. The outcome is unique and:

(a) if $\left(\bar{p}^{s}, \bar{p}^{b}\right) \in I R\left(P_{t}\right)$, then the outcome is trade at $\hat{p}_{t}\left(\bar{p}^{s}, \bar{p}^{b}\right)$;

(b) if $\left(\bar{p}^{s}, \bar{p}^{b}\right) \notin I R\left(P_{t}\right)$, then the outcome is no trade.

Proof of Claim 1: The set of possible outcomes from the above mechanism is $P_{t}$. Thus (b) follows by the same logic as the lemma, noting that in this case a subgame perfect equilibrium exists because no price is ever approved by both agents. Similarly, if $\left(\bar{p}^{s}, \bar{p}^{b}\right) \in \operatorname{IR}\left(P_{t}\right)$, then the unique subgame perfect equilibrium outcome is trade at some price, provided an equilibrium exists. We need to show that a subgame perfect equilibrium exists and it is trade at $\hat{p}_{t}\left(\bar{p}^{b}, \bar{p}^{s}\right)$.

Consider the following strategies which result in $\hat{p}_{t}\left(\bar{p}^{b}, \bar{p}^{s}\right)$. It is easily checked that given these expectations, these form a subgame perfect equilibrium.

On the equilibrium path behavior: The seller announces $p^{s}=\bar{p}^{s}$. The buyer announces $\left(p^{s^{\prime}}, p^{b}\right)$ $=\left(\bar{p}^{s}, \bar{p}^{b}\right)$. The seller announces $p^{b^{\prime}}=\bar{p}^{b}$. Both approve this.

Off the equilibrium path behavior:

Each player approves any price that is individually rational, and vetos others.

If the seller announces $p^{s}<\bar{p}^{s}$, then the buyer announces $\left(p^{s^{\prime}}, p^{b}\right)=\left(p^{s}, \bar{p}^{b}\right)$.

If the seller announces $p^{s}<\bar{p}^{s}$, then the buyer announces $\left(p^{s^{\prime}}, p^{b}\right)=\left(p^{s}, \bar{p}^{b}\right)$.

If the buyer announces $p^{b}<\bar{p}^{b}$, then the seller announces $p^{b^{\prime}}=\bar{p}^{b}$.

To see that this is an equilibrium, notice first that if the seller announces a price in excess of his reservation price, the buyer can correct the announcement and win all the surplus, and (by (V5)) end up paying a lower price. If the seller announces a price below his reservation price, he is directly 
conceding some surplus to the buyer. If the seller tells the truth, then the buyer cannot claim the seller has a lower reservation price, or this will lead to no trade. Similarly, the buyer cannot gain from understating his reservation price, since the seller could then correct this announcement to the true buyer reservation value, and win all the surplus.

Q.E.D.

\section{ClaIM 2: There exists an equilibrium which results in $\tilde{A}_{t}, \tilde{p}_{t}$.}

Proof of Claim 2: If we fix the reservation prices of the buyers and sellers, then there is a unique subgame perfect equilibrium outcome for any matched pair for a specific stage. So fix the reservation prices at those generated by the allocation rule, $\tilde{A}_{t}, \tilde{p}_{t}$. We will verify that the subgame perfect equilibrium outcome in this case results in $\tilde{A}_{t}, \tilde{p}_{t}$.

If $(s, b) \notin \tilde{A}_{t}$, then no trade is the only subgame perfect outcome of the augmented mechanism. This follows from (V3) and Claim 1.

If $(s, b) \in \tilde{A}_{t}$, then from Claim 1 and (V1) and (V2) it follows that the outcome is trade at $\hat{p}_{t}\left(\bar{p}_{t}^{s}(s ; A, p), \bar{p}_{t}^{b}(b ; A, p)\right)$.

Q.E.D.

ClaIM 3: If $\left(A^{\prime}, p^{\prime}\right)$ is not equivalent to $(A, p)$, then $\left(A^{\prime}, p^{\prime}\right)$ is not the result of any equilibrium of the mechanism.

Proof of Claim 3: Suppose to the contrary that there is an equilibrium that results in $\left(A^{\prime}, p^{\prime}\right)$.

Consider $(s, b) \notin A_{t}^{\prime}$. It must be that the outcome of $\Gamma\left(\gamma_{t}\right)$ is no trade. From Claim 1, it then follows that (V3) holds relative to $\hat{p}_{t}$ and $P_{t}$.

Consider $(s, b) \in A_{t}^{\prime}$. It must be that the outcome of $\Gamma\left(\gamma_{t}\right)$ is trade at $p_{t}^{\prime}(s, b)$. It then follows from Claim 1 that (V1) and (V2) hold relative to $\hat{p}_{t}$ and $P_{t}$.

This contradicts (ii), which implies that $\left(A^{\prime}, p^{\prime}\right)$ fails to satisfy (V1), (V2), or (V3) relative to $\hat{p}_{t}$ and $P_{t}$.

Q.E.D.

After Theorem 4, we claimed that (i) would be necessary even if one only considers attainability, and also if one drops the requirement of a closed mechanism. This is the same as the above proof of the necessity of (i), except that lemma is only stated for $\left(\bar{p}^{s}, \bar{p}^{b}\right)$ relative to which equilibrium exists. Then one needs to extend $\hat{p}_{t}$ to satisfy (V2), (V4), and (V5), for $\left(\bar{p}^{s}, \bar{p}^{b}\right) \in I R\left(P_{t}\right)$ relative to which there does not exist an equilibrium. For such a $\left(\bar{p}^{s}, \bar{p}^{b}\right)$, define $\hat{p}_{t}\left(\bar{p}^{s}, \bar{p}^{b}\right)$ by setting it equal to the $\max$ of $\bar{p}^{s}$ and the sup of $\hat{p}_{t}$ over $\left(\bar{p}^{s^{\prime}}, \bar{p}^{b^{\prime}}\right) \in \operatorname{IR}\left(P_{t}\right)$ such that $\bar{p}^{s^{\prime}} \leq \bar{p}^{s}, \bar{p}^{b^{\prime}} \leq \bar{p}^{b}$, and for which there exists an equilibrium. This construction satisfies (V2), (V4), and (V5).

Q.E.D.

Proof of Theorem 2: This follows directly from Theorem 4 and the proof above.

Q.E.D.

Proof of Theorem 3: It follows from Theorem 4 (and the proof above and the continuity so that if any equivalent allocation rule satisfies strong individual rationality and has nondecreasing prices, then $(A, p)$ does as well) that the conditions are necessary. To see that they are sufficient wa: show that the voluntary trade condition of Theorem 4 is satisfied relative to $(A, p)$. Then the result follows from Claims 1 and 2 in the proof of Theorem 4.

Under the assumptions of Theorem 3, reservation prices are continuous and nondecreasing functions of $s$ and $b$. Given the continuity of $p$ and $A$ it follows that individual rationality must hold with exact equality for cutoff pairs. ${ }^{33}$ Thus, if $b=\beta_{t}(s)$ (where $\beta_{t}$ is the cutoff defined by the cutoff rule $\left.A_{t}\right)$, then $\bar{p}_{t}(b)=\bar{p}_{t}(s)=p_{t}(s, b)$. To see this, consider a cutoff pair $s, b$. By individual rationality $\bar{p}_{t}(b) \geq p_{t}(s, b) \geq \bar{p}_{t}(b)$. For any $b^{\prime}<b$ we know that either $\bar{p}_{t}\left(b^{\prime}\right)<p_{t}(s, b)$ or $\bar{p}_{t}(s)>p_{t}(s, b)$. We know that the second cannot hold, so it must be that $\bar{p}_{t}\left(b^{\prime}\right)<p_{t}(s, b)$. Then by continuity, $\bar{p}_{t}(b)=p_{t}(s, b)$. Similar reasoning establishes $\bar{p}_{t}(s)=p_{t}(s, b)$.

${ }^{33}$ Given the continuity, all claims that were "almost every," no longer have that qualifier. 
Let $\underline{s}_{t}$ be the $\min \left\{s \mid(s, b) \in A_{t}\right.$ for some $\left.b\right\}$, and $\bar{s}_{t}$ be the $\max \left\{s \mid(s, b) \in A_{t}\right.$, for some $\left.b\right\}$. Similarly define $\underline{b}_{t}$ and $\bar{b}_{t}$. Next, notice that $\bar{p}_{t}\left(\underline{s}_{t}\right)=\bar{p}_{t}\left(\underline{b}_{t}\right)=p_{t}\left(\underline{s}_{t}, \underline{b}_{t}\right)$ and similarly, $\bar{p}_{t}\left(\bar{s}_{t}\right)=\bar{p}_{t}\left(\bar{b}_{t}\right)=p_{t}\left(\bar{s}_{t}, \bar{b}_{t}\right)$. Given the assumptions on $A_{t}$ and $p_{t}$, the range of $p_{t}$ over pairs in $A_{t}$ is $\left[p_{t}\left(\underline{s}_{t}, \underline{b}_{t}\right), p_{t}\left(\bar{s}_{t}, \bar{b}_{t}\right)\right]$, since $\left(\underline{s}_{t}, \underline{b}_{t}\right) \in A_{t}$ and $\left(\bar{s}_{t}, \bar{b}_{t}\right) \in A_{t}$ given that $A_{t}$ is a cutoff rule and cutoffs are nondecreasing in value. So, let $P_{t}=\left[p_{t}\left(\underline{s}_{t}, \underline{b}_{t}\right), p_{t}\left(\bar{s}_{t}, \bar{b}_{t}\right)\right]$. For $\bar{p}^{s}, \bar{p}^{b} \in \operatorname{IR}\left(P_{t}\right)$ define $\hat{p}_{t}\left(\bar{p}^{s}, \bar{p}^{b}\right)$ through $p_{t}(s, b)$ by setting

$$
\hat{p}_{t}\left(\bar{p}^{s}, \bar{p}^{b}\right)=p_{t}\left(s^{\prime}, b^{\prime}\right)
$$

where $s^{\prime}=\min \left\{s \mid \bar{p}_{t}^{s}(s) \geq \max \left\{\bar{p}^{s}, \bar{p}_{t}^{s}\left(\underline{s}_{t}\right)\right\}\right\}$ and $b^{\prime}=\max \left\{b \mid \bar{p}_{t}^{s}(b) \geq \min \left\{\bar{p}^{b}, \bar{p}_{t}^{b}\left(\bar{b}_{t}\right)\right\}\right\}$. Using this we verify that the voluntary trade condition is satisfied relative to $(A, p)$. (V1) holds since if $s, b \in A_{t}$, then $s^{\prime}=s$ and $b^{\prime}=b$ in our definition above. (V2) holds by the construction of $\hat{p}_{t}$ and the individual rationality assumed in Theorem 3 . (V3) holds by the strong individual rationality assumed in Theorem 3. (V4) holds by the construction of $\hat{p}_{t}$. To see (V5), notice that for $\hat{p}_{t}\left(\bar{p}^{s^{\prime}}, \bar{p}^{b}\right)<\hat{p}_{t}\left(\bar{p}^{s}, \bar{p}^{b}\right)$, it must be that $\min \left\{s \mid \bar{p}_{t}^{s}(s) \geq \max \left\{\bar{p}^{s^{\prime}}, \bar{p}_{t}^{s}\left(\underline{s}_{t}\right)\right\}\right\}<\min \left\{s \mid \bar{p}_{t}^{s}(s) \geq \max \left\{\bar{p}^{s}, \bar{p}_{t}^{s}\left(\underline{s}_{t}\right)\right\}\right\}$. Thus, from the definition of $\hat{p}$, there exists $s^{\prime}<s$ and $b$ with $\hat{p}_{t}\left(\bar{p}^{s^{\prime}}, \bar{p}^{b}\right)=p_{t}\left(s^{\prime}, b\right)$ and $\hat{p}_{t}\left(\bar{p}^{s}, \bar{p}^{b}\right)=p_{t}(s, b)$. Given the individual rationality in Theorem 3 , we know that $\left(s^{\prime}, b\right) \in A_{t}$ and $(s, b) \in A_{t}$. By continuity of $p$, we can find $s^{\prime \prime}, b \in A_{t}$ with $p_{t}\left(s^{\prime}, b\right)<p_{t}\left(s^{\prime \prime}, b\right)<p_{t}(s, b)$. Then (V5) is satisfied with $p=p_{t}\left(s^{\prime \prime}, b\right)$. The same can be done for the other part of the condition concerning buyer values.

Q.E.D.

Proof of Proposition 1: Consider distributions such as those described after Proposition 1.

From Theorem 1, the cutoff rule for the efficient allocation for the seller with $s=0^{34}$ satisfies

$$
\beta_{1}(0)=E_{2}(b)+E_{2}\left(\max \left[\beta_{1}(0)-s, 0\right]\right)-E_{2}(\max [b-s, 0]) .
$$

As $\epsilon$ becomes small, $E_{2}(b)$ converges to $\left(B_{2}(1)-B_{2}(.5)\right) / B_{2}(1), E_{2}\left(\max \left[\beta_{1}(0)-s, 0\right]\right)$ converges to $B_{1}(0) S_{2}(.5) / S_{2}(1)$ and $E_{2}(\max [b-s, 0])$ converges to $\left(B_{2}(1)-B_{2}(.5)\right) S_{2}(.5) /\left(S_{2}(1) B_{2}(1)\right)$. So at the limit (as $\epsilon$ becomes small),

$$
\beta_{1}(0)=\frac{B_{2}(1)-B_{2}(.5)}{B_{2}(1)}+\frac{\beta_{1}(0) S_{2}(.5)}{S_{2}(1)}-\frac{\left(B_{2}(1)-B_{2}(.5)\right) S_{2}(.5)}{S_{2}(1) B_{2}(1)} .
$$

Solving for $\beta_{1}(0)$

$$
\beta_{1}(0)=\frac{B_{2}(1)-B_{2}(.5)}{B_{2}(1)}
$$

This means that $\beta_{1}(0)$ is at most $1 / 2$, since none of the $b=0$ 's are cleared in the first period. So, as $\epsilon$ goes to zero, it must be that $B_{2}(.5)=1 / 2$ and $B_{2}(1)=3 / 4$. Thus, $\beta_{1}(0)$ converges to $1 / 3$ as $\epsilon$ goes to zero. ${ }^{35}$ Similarly, the cutoff for buyers with a valuation of $1, \sigma_{1}(1)$, converges to $2 / 3$ as $\epsilon$ goes to zero.

Let $A$ be equivalent to the efficient trading rule and consider any price rule $p$. We show that for small enough $\epsilon$ the necessary conditions for voluntary attainability cannot be satisfied for $(A, p)$. Suppose to the contrary that they are for all small $\epsilon$. Pick some small $\gamma>0$, and apply strong individual rationality to $b^{\prime}=\beta_{1}(0)-\gamma$ and $s^{\prime}=0$. Since we know that individual rationality is satisfied for $s$ close to 0 and some $b$ close to $\beta_{1}(0)$, it follows that for some small enough $\gamma^{36}$ $\beta_{1}(0)-\gamma$ should not trade in period 1 so that

$$
\beta_{1}(0)-\gamma-p_{1}\left(0, \beta_{1}(0)\right)<\frac{S_{2}(.5)}{S_{2}(1)}\left(\beta_{1}(0)-\gamma-p_{2}\left(\gamma, \beta_{1}(0)-\gamma\right)\right)+2 \epsilon
$$

${ }^{34}$ Since the efficient solution is only defined up to sets of measure 0 , one can find $s$ close to 0 and work with that.

${ }^{35}$ Uniqueness of the efficient solution and continuity of the welfare function in changes in distribution of buyer and sellers' values imply continuity of the efficient solution in the distribution of buyer and sellers' values.

${ }^{36}$ We proceed as if this is satisfied for $s=0$ and $b=\beta_{1}(0)$, while for any trading rule equivalent to the efficient one this can be redefined for some $s, b$ arbitrarily close to these. 
where the right-hand side bounds the expected value to rematching: there is a probability of at most $S_{2}(.5) / S_{2}(1)$ that the buyer is matched with someone with a value between $\gamma$ and $\frac{1}{2}$, and the best price they can get is then $p_{2}\left(\gamma, \beta_{1}(0)-\gamma\right)$. For $\gamma$ small enough, there is at most $2 \epsilon$ chance that they are matched with a seller with value smaller than $\gamma$.

A similar argument for $s^{\prime}=\gamma$ and $b^{\prime}=\beta_{1}(0)$ leads to

$$
p_{1}\left(0, \beta_{1}(0)\right)-\gamma<\frac{B_{2}(1)-B_{2}(.5)}{B_{2}(1)}\left(p_{2}(\gamma, 1-\gamma)-\gamma\right)+2 \epsilon .
$$

Given the symmetry of the distributions and thus the efficient solution,

$$
\frac{S_{2}(.5)}{S_{2}(1)}=\frac{B_{2}(1)-B_{2}(.5)}{B_{2}(1)} \text {. }
$$

Summing the two previous inequalities and simplifying leads to

$$
\left(\beta_{1}(0)-2 \gamma\right)\left(1-\frac{S_{2}(.5)}{S_{2}(1)}\right)<\frac{S_{2}(.5)}{S_{2}(1)}\left(p_{2}(\gamma, 1-\gamma)-p_{2}\left(\gamma, \beta_{1}(0)-\gamma\right)\right)+4 \epsilon .
$$

We can follow the same arguments in a neighborhood of the buyer with value 1 and that buyer's cutoff seller, $\sigma_{1}(1)$, to find that

$$
\left(1-\sigma_{1}(1)-2 \gamma\right)\left(1-\frac{S_{2}(.5)}{S_{2}(1)}\right)<\frac{S_{2}(.5)}{S_{2}(1)}\left(p_{2}\left(\sigma_{1}(1)+\gamma, 1-\gamma\right)-p_{2}(\gamma, 1-\gamma)\right) 4 \epsilon .
$$

Summing these two inequalities, for small $\epsilon$ and $\gamma$ this is approximately

$$
\left(\beta_{1}(0)+1-\sigma_{1}(1)\right)\left(1-\frac{S_{2}(.5)}{S_{2}(1)}\right)<\frac{S_{2}(.5)}{S_{2}(1)}\left(p_{2}\left(\sigma_{1}(1)-\gamma, 1-\gamma\right)-p_{2}\left(\gamma, \beta_{1}(0)-\gamma\right)\right)
$$

or

$$
\frac{4}{9}<\frac{1}{3}\left(p_{2}\left(\sigma_{1}(1)+\gamma, 1-\gamma\right)-p_{2}\left(\gamma, \beta_{1}(0)-\gamma\right)\right)
$$

which is impossible to satisfy since the right-hand side is at most $1 / 3$.

Q.E.D.

\section{REFERENCES}

Aghion, P., M. Dewatripont, ANd P. Rey (1994): "Renegotiation Design with Unverifiable Information," Econometrica, 62, 257-282.

ABREU, D., AND H. MATSUSHIMA (1992): "Virtual Implementation in Iterative Undominated Strategies: Complete Information," Econometrica, 60, 993-1008.

AL-NAJJAR, N. (1996): “Aggregation and the Law of Large Numbers in Economies with a Continuum of Agents," Mimeo, Northwestern University.

BALIGA, S., L. CORChON, AND T. SJöström (1997): "The Theory of Implementation when the Planner is a Player," Journal of Economic Theory, 77, 15-33.

BALIGA, S., AND T. SJÖSTRÖM (1995): "Interactive Implementation,” Mimeo, Harvard University.

BinMore, K., AND M. HerRero (1988): "Matching and Bargaining in Dynamic Markets," Review of Economic Studies, 55, 17-32.

BRusco, S., AND M. JACKSON (1996): "The Optimal Design of Security Markets," Mimeo, Northwestern University.

Butters, G. (1980): “Equilibrium Price Distributions in a Random Meetings Market,” Mimeo, Princeton University.

Chakravorti, B., L. CoRchon, AND S. Wilkie (1992): “Credible Implementation,” forthcoming in Games and Economic Behavior.

DAsGupta, P., P. HAMmond, AND E. MASkin (1979): "The Implementation of Social Choice Rules: Some General Results on Incentive Compatibility," Review of Economic Studies, 46, 185-216. 
Diamond, P. (1982): "Wage Determination and Efficiency in Search Equilibrium," Review of Economic Studies, 49, 217-227.

DutTA, B., A. SEN, AND R. Vohra (1995): "Nash Implementation Through Elementary Mechanisms in Economic Environments," Economic Design, 1, 173-204.

Feldman, M., AND C. GILles (1985): "An Expository Note on Individual Risk without Aggregate Uncertainty," Journal of Economic Theory, 35, 26-32.

Gale, D. (1986a): "Bargaining and Competition Part I: Characterization," Econometrica, 54, 785-806.

- (1986b): "Bargaining and Competition Part II: Existence," Econometrica, 54, 807-818.

Gretsky, N., J. Ostroy, AND W. Zame (1992): "The Nonatomic Assignment Model," Economic Theory, 2, 103-127.

HART, O., AND J. MoORE (1988): "Incomplete Contracts and Renegotiation," Econometrica, 56, 755-785.

Herrero, M., AND S. SRIVAStava (1992): "Implementation via Backward Induction," Journal of Economic Theory, 56, 70-88.

Hosios, A. (1990): "On the Efficiency of Matching and Related Models of Search and Unemployment," Review of Economic Studies, 57, 279-298.

Hurwicz, L. (1972): "On Informationally Decentralized Systems," in Decision and Organization, ed. by C. B. McGuire and R. Radner. Amsterdam: North Holland.

JACKSON, M. (1992): "Implementation in Undominated Strategies: A Look at Bounded Mechanisms," Review of Economic Studies, 59, 757-775.

JACKSON, M., AND T. PALFREY (1997): "Efficiency and Voluntary Implementation in Markets with Repeated Pairwise Bargaining," Social Science Working Paper \#985, California Institute of Technology.

Jacksan, M., T. Palfrey, and S. Srivastava (1994): "Undominated Nash Implementation in Bounded Mechanisms," Games and Economic Behavior, 6, 474-501.

JuDD, K. (1985): "The Law of Large Numbers with a Continuum of I.I.D. Random Variables," Journal of Economic Theory, 35, 19-25.

KALAI, E., AND J. LEDYARD (1995): "Repeated Implementation," Mimeo, California Institute of Technology.

Lu, X., AND P. McAfEe (1995): "Matching and Expectations in a Market with Heterogeneous Agents," in Advances in Applied Microeconomics, ed. by M. Baye. Connecticut: JAI Press.

MA, C., J. Moore, AND S. TuRnbull (1988): "Stopping Agents from Cheating," Journal of Economic Theory, 46, 355-372.

MASKIN, E., AND J. MoORE (1987): “Implementation and Renegotiation," Mimeo, Harvard University.

MCLENNAN, A., AND H. SonNENSCHIEN (1991): "Sequential Bargaining as a Noncooperative Foundation for Walrasian Equilibria," Econometrica, 59, 1395-1424.

MoORE, J. (1992): "Implementation, Contracts, and Renegotiation Environments with Complete Information," in Advances in Economic Theory, ed. by J.-J. Laffont. Cambridge: Cambridge University Press, pp. 182-282.

Mortensen, D. (1982): "The Matching Process as a Noncooperative Bargaining Game," in The Economics of Information and Uncertainty, ed. by J. J. McCall. Chicago: University of Chicago Press.

Peters, M. (1991): "Ex Ante Price Offers in Matching Games Non-Steady States," Econometrica, $59,1424-1444$.

Ponsati, C., AND J. SAKovics (1995): "Rubinstein Bargaining with Two-Sided Outside Options," Mimeo, Universitat Autonoma de Barcelona.

Postlewaite, A., AND D. WetTstein (1989): "Continuous and Feasible Implementation," Review of Economic Studies, 56, 14-33.

RubinsteIn, A. (1982): "Perfect Equilibrium in a Bargaining Model," Econometrica, 50, 97-109.

RubENSTEIN, A., AND A. Wolinsky (1985): "Equilibrium in a Market with Sequential Bargaining," Econometrica, 53, 1133-1150. 
(1990): "Renegotiation-Proof Implementation," WP14-90, Tel Aviv University.

(1992): "Renegotiation-Proof Implementation and Time Preferences," American Economic Review, 82, 600-614.

SAiJO, T., Y. TATimatani, AND T. Yamato (1993): "Toward Natural Implementation," forthcoming in International Economic Review.

SATtinger, M. (1995): "Search and the Efficient Assignment of Workers to Jobs," International Economic Review, 36, 283-302.

Shimer, D., AND L. SMith (1994): "The Normative Implications of Heterogeneity in Search," Mimeo, MIT.

(1996): "Assortative Matching and Search," Mimeo, MIT.

SJöströM, T. (1995): "Implementation by Demand Mechanisms," Economic Design, 1, 343-354.

STAHL, I. (1972): Bargaining Theory. Stockholm: Stockholm School of Economics. 